\title{
On Perturbative Cubic Nonlinear Schrodinger Equations under Complex Nonhomogeneities and Complex Initial Conditions
}

\author{
Magdy A. El-Tawil ${ }^{1}$ and Maha A. El-Hazmy² \\ ${ }^{1}$ Department of Engineering Mathematics, Faculty of Engineering, Cairo University, Giza 12613, Egypt \\ ${ }^{2}$ Department of Mathematics, Girls College, Medina, Saudi Arabia
}

Correspondence should be addressed to Magdy A. El-Tawil, magdyeltawil@yahoo.com

Received 12 February 2009; Revised 30 May 2009; Accepted 8 July 2009

Recommended by Roger Grimshaw

A perturbing nonlinear Schrodinger equation is studied under general complex nonhomogeneities and complex initial conditions for zero boundary conditions. The perturbation method together with the eigenfunction expansion and variational parameters methods are used to introduce an approximate solution for the perturbative nonlinear case for which a power series solution is proved to exist. Using Mathematica, the symbolic solution algorithm is tested through computing the possible approximations under truncation procedures. The method of solution is illustrated through case studies and figures.

Copyright (C) 2009 M. A. El-Tawil and M. A. El-Hazmy. This is an open access article distributed under the Creative Commons Attribution License, which permits unrestricted use, distribution, and reproduction in any medium, provided the original work is properly cited.

\section{Introduction}

The nonlinear Schrodinger (NLS) equation is the principal equation to be analyzed and solved in many fields, see [1-5], for examples. In the last two decades, there are a lot of NLS problems depending on additive or multiplicative noise in the random case $[6,7]$ or a lot of solution methodologies in the deterministic case.

Wang et al. [8] obtained the exact solutions to NLS using what they called the subequation method. They got four kinds of exact solutions of

$$
i \frac{\partial u}{\partial t}+\frac{1}{2} \frac{\partial^{2} u}{\partial x^{2}}+\alpha|u|^{p} u+\beta|u|^{2 p} u=0
$$

for which no sign to the initial or boundary conditions type is made. $\mathrm{Xu}$ and Zhang [9] 
followed the same previous technique in solving the higher-order NLS:

$$
i \frac{\partial u}{\partial x}-\frac{1}{2} \alpha \frac{\partial^{2} u}{\partial t^{2}}+\beta|u|^{2} u+i \varepsilon \frac{\partial^{3} u}{\partial t^{3}}+i \delta|u|^{2} \frac{\partial u}{\partial t}+i \gamma u^{2} \frac{\partial u^{*}}{\partial t}=0 .
$$

Sweilam [10] solved

$$
i \frac{\partial u}{\partial t}+\frac{\partial^{2} u}{\partial x^{2}}+q|u|^{2} u=0, \quad t>0, L_{0}<x<L_{1}
$$

with initial condition $u(x, 0)=g(x)$ and boundary conditions $u_{x}\left(L_{0}, t\right)=u_{x}\left(L_{1}, t\right)=0$, which gives rise to solitary solutions using variational iteration method. Zhu [11] used the extended hyperbolic auxiliary equation method in getting the exact explicit solutions to the higherorder NLS:

$$
i q_{z}-\frac{\beta_{1}}{2} q_{t t}+\gamma_{1}|q|^{2} q=i \frac{\beta_{2}}{6} q_{t t t}+\frac{\beta_{3}}{24} q_{t t t t}-\gamma_{2}|q|^{4} q
$$

without any conditions. Sun et al. [12] solved the NLS:

$$
i \frac{\partial \psi}{\partial t}+\frac{\partial^{2} \psi}{\partial x^{2}}+a|\psi|^{2} \psi=0
$$

with the initial condition $\psi(x, 0)=\psi_{0}(x)$ using Lie group method. By using coupled amplitude phase formulation, Porsezian and Kalithasan [13] constructed the quartic anharmonic oscillator equation from the coupled higher-order NLS. Two-dimensional grey solitons to the NLS were numerically analyzed by Sakaguchi and Higashiuchi [14]. The generalized derivative NLS was studied by Huang et al. [15] introducing a new auxiliary equation expansion method. Abou Salem and Sulem [16] studied the effective dynamics of solitons for the generalized Schrodinger equation in a random potential. El-Tawil [17] considered a nonlinear Schrodinger equation with random complex input and complex initial conditions. Colin et al. [18] considered three components of nonlinear Schrodinger equations related to the Raman amplification in a plasma. In [19], Jia-Min and Yu-Lu constructed an appropriate transformations and an extended elliptic subequation approach to find some exact solutions for variable coefficient cubic-quintic nonlinear Schrodinger equation with an external potential.

In this paper, a straight forward solution algorithm is introduced using the transformation from a complex solution to a coupled equations in two real solutions, eliminating one of the solutions to get separate independent and higher-order equations, and finally introducing a perturbative approximate solution to the system.

\section{The Linear Case}

Consider the nonhomogeneous linear Schrodinger equation:

$$
i \frac{\partial u(t, z)}{\partial z}+\alpha \frac{\partial^{2} u(t, z)}{\partial t^{2}}=F_{1}(t, z)+i F_{2}(t, z), \quad(t, z) \in(0, T) \times(0, \infty),
$$


where $u(t, z)$ is a complex valued function which is subjected to

$$
\begin{array}{ll}
\text { I.C. : } & u(t, 0)=f_{1}(t)+i f_{2}(t), \text { a complex valued function, } \\
\text { B.C. : } & u_{z}(0, z)=0, \quad u_{z}(T, z)=0 .
\end{array}
$$

Let $u(t, z)=\psi(t, z)+i \phi(t, z), \psi, \phi$ : real valued functions. Substituting (2.2) in (2.1), the following coupled equations are got as follows:

$$
\begin{aligned}
& \frac{\partial \phi(t, z)}{\partial z}=\alpha \frac{\partial^{2} \psi(t, z)}{\partial t^{2}}+G_{1}(t, z), \\
& \frac{\partial \psi(t, z)}{\partial z}=\alpha \frac{\partial^{2} \phi(t, z)}{\partial t^{2}}+G_{2}(t, z),
\end{aligned}
$$

where $\psi(t, 0)=f_{1}(t), \phi(t, 0)=f_{2}(t), G_{1}(t, z)=-F_{1}(t, z), G_{2}(t, z)=F_{2}(t, z)$, and all corresponding other I.C. and B.C. are zeros. equations:

Eliminating one of the variables in (2.3), one can get the following independent

$$
\begin{aligned}
& \frac{\partial^{4} \psi(t, z)}{\partial t^{4}}+\frac{1}{\alpha^{2}} \frac{\partial^{2} \psi(t, z)}{\partial z^{2}}=\frac{1}{\alpha^{2}} \widetilde{\psi}_{1}(t, z) \\
& \frac{\partial^{4} \phi(t, z)}{\partial t^{4}}+\frac{1}{\alpha^{2}} \frac{\partial^{2} \phi(t, z)}{\partial z^{2}}=\frac{1}{\alpha^{2}} \widetilde{\psi}_{2}(t, z)
\end{aligned}
$$

where

$$
\begin{aligned}
& \tilde{\psi}_{1}(t, z)=\frac{\partial G_{2}}{\partial z}-\alpha \frac{\partial^{2} G_{1}}{\partial t^{2}} \\
& \tilde{\psi}_{2}(t, z)=\alpha \frac{\partial G_{2}}{\partial t^{2}}+\frac{\partial G_{1}}{\partial z} .
\end{aligned}
$$

Using the eigenfunction expansion technique [20], the following solutions for (2.4) are obtained:

$$
\begin{aligned}
& \psi(t, z)=\sum_{n=0}^{\infty} T_{n}(z) \sin \left(\frac{n \pi}{T}\right) t \\
& \phi(t, z)=\sum_{n=0}^{\infty} \tau_{n}(z) \sin \left(\frac{n \pi}{T}\right) t
\end{aligned}
$$

where $T_{n}(z)$ and $\tau_{n}(z)$ can be got through the applications of initial conditions and then solving the resultant second-order differential equations using the method of the variational 
parameter [21]. The final expressions can be got as follows

$$
\begin{aligned}
& T_{n}(z)=\left(C_{1}+A_{1}(z)\right) \sin \beta_{n} z+\left(C_{2}+B_{1}(z)\right) \cos \beta_{n} z \\
& \tau_{n}(z)=\left(C_{3}+A_{2}(z)\right) \sin \beta_{n} z+\left(C_{4}+B_{2}(z)\right) \cos \beta_{n} z
\end{aligned}
$$

where

$$
\begin{aligned}
\beta_{n} & =\alpha\left(\frac{n \pi}{T}\right)^{2}, \\
A_{1}(z) & =\frac{1}{\beta_{n}} \int \tilde{\psi}_{1 n}(z ; n) \cos \left(\beta_{n}\right) z d z, \\
B_{1}(z) & =\frac{-1}{\beta_{n}} \int \tilde{\psi}_{1 n}(z ; n) \sin \left(\beta_{n}\right) z d z, \\
A_{2}(z) & =\frac{1}{\beta_{n}} \int \tilde{\psi}_{2 n}(z ; n) \cos \left(\beta_{n}\right) z d z, \\
B_{2}(z) & =\frac{-1}{\beta_{n}} \int \tilde{\psi}_{2 n}(z ; n) \sin \left(\beta_{n}\right) z d z,
\end{aligned}
$$

in which

$$
\begin{aligned}
& \tilde{\psi}_{1 n}(z ; n)=\frac{2}{T} \int_{0}^{T} \tilde{\psi}_{1}(t, z) \sin \left(\frac{n \pi}{T} t\right) d t \\
& \tilde{\psi}_{2 n}(z ; n)=\frac{2}{T} \int_{0}^{T} \tilde{\psi}_{2}(t, z) \sin \left(\frac{n \pi}{T} t\right) d t
\end{aligned}
$$

The following conditions should also be satisfied:

$$
\begin{aligned}
& C_{2}=\frac{2}{T} \int_{0}^{T} f_{1}(t) \sin \left(\frac{n \pi}{T}\right) d t-B_{1}(0), \\
& C_{4}=\frac{2}{T} \int_{0}^{T} f_{2}(t) \sin \left(\frac{n \pi}{T}\right) d t-B_{2}(0) .
\end{aligned}
$$

Finally, the following solution is obtained:

$$
u(t, z)=(\psi(t, z)+i \phi(t, z))
$$

or

$$
|u(t, z)|^{2}=\left(\psi^{2}(t, z)+\phi^{2}(t, z)\right)
$$




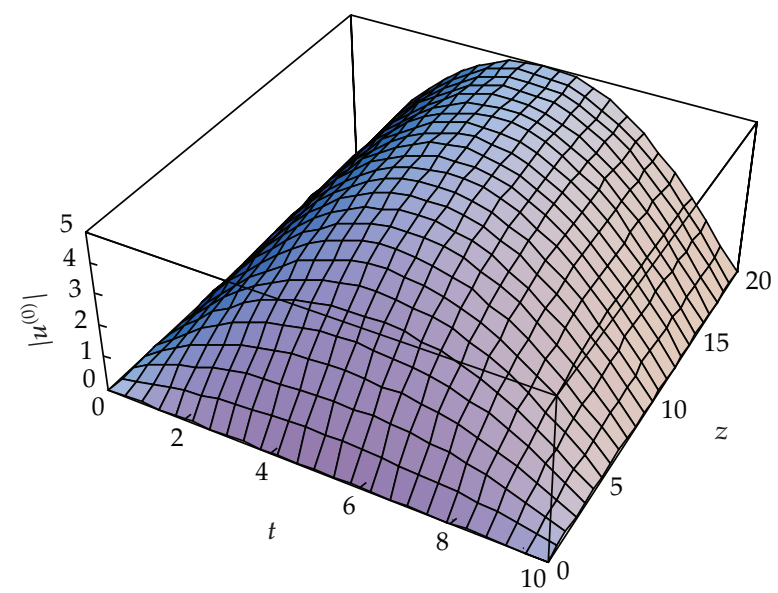

Figure 1: The zero-order approximation of $\left|u^{(0)}\right|$ at $\alpha=1, T=10, \gamma=02$ with considering only one term in the series $(M=1)$.

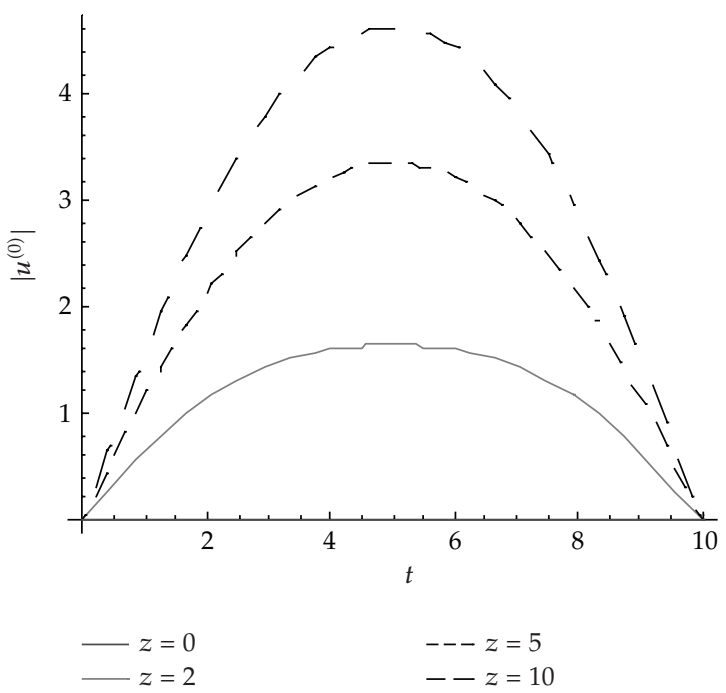

Figure 2: The zero-order approximation $\left|u^{(0)}\right|$ at $\alpha=1, T=10, \gamma=02$ for different values of $z$, considering only one term in the series $(M=1)$.

\section{The Nonlinear Case}

Consider the homogeneous nonlinear Schrodinger equation:

$$
\begin{array}{r}
i \frac{\partial u(t, z)}{\partial z}+\alpha \frac{\partial^{2} u(t, z)}{\partial t^{2}}+\varepsilon|u(t, z)|^{2} u(t, z)+i \gamma u(t, z)=F_{1}(t, z)+i F_{2}(t, z), \\
(t, z) \in(0, T) \times(0, \infty),
\end{array}
$$

where $u(t, z)$ is a complex-valued function which is subjected to the initial and boundary conditions (2.2). 


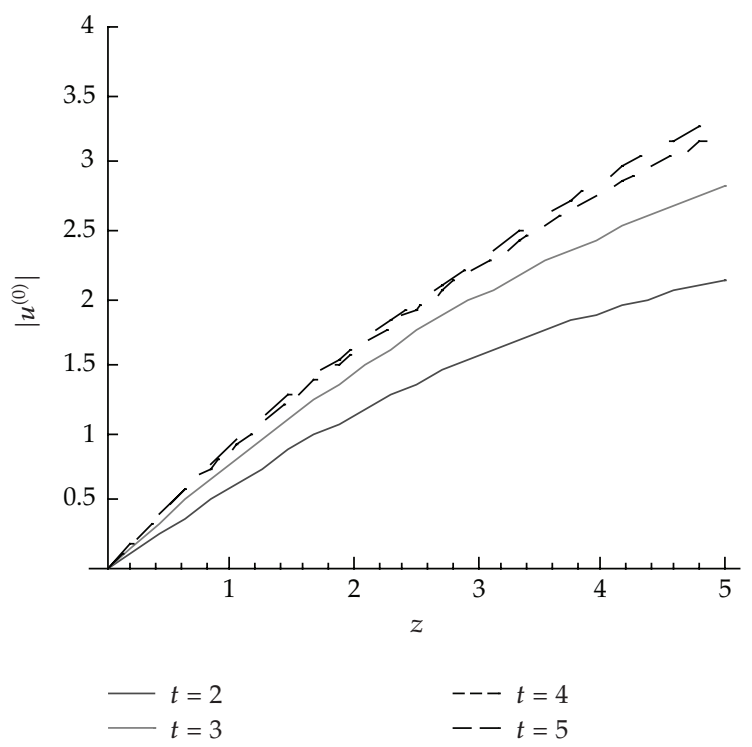

Figure 3: The zero-order approximation $\left|u^{(0)}\right|$ at $\alpha=1, T=10, \gamma=02$ for different values of $t$, considering only one term in the series $(M=1)$.

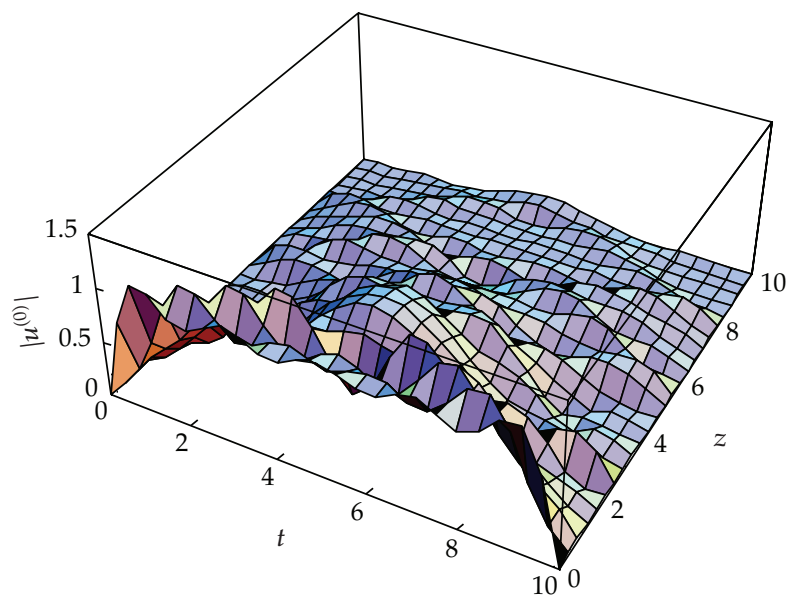

Figure 4: The zero-order approximation of $\left|u^{(0)}\right|$ at $\alpha=1, T=10, \gamma=02$ with considering only one term in the series $(M=1)$.

Lemma 3.1. The solution of (3.1) with the constraints (2.2) is a power series in $\varepsilon$ if the solution exists.

Proof. At $\varepsilon=0$, the following linear equation is got:

$$
i \frac{\partial u(t, z)}{\partial z}+\alpha \frac{\partial^{2} u(t, z)}{\partial t^{2}}+i \gamma u(t, z)=F(t, z), \quad(t, z) \in(0, T) \times(0, \infty),
$$




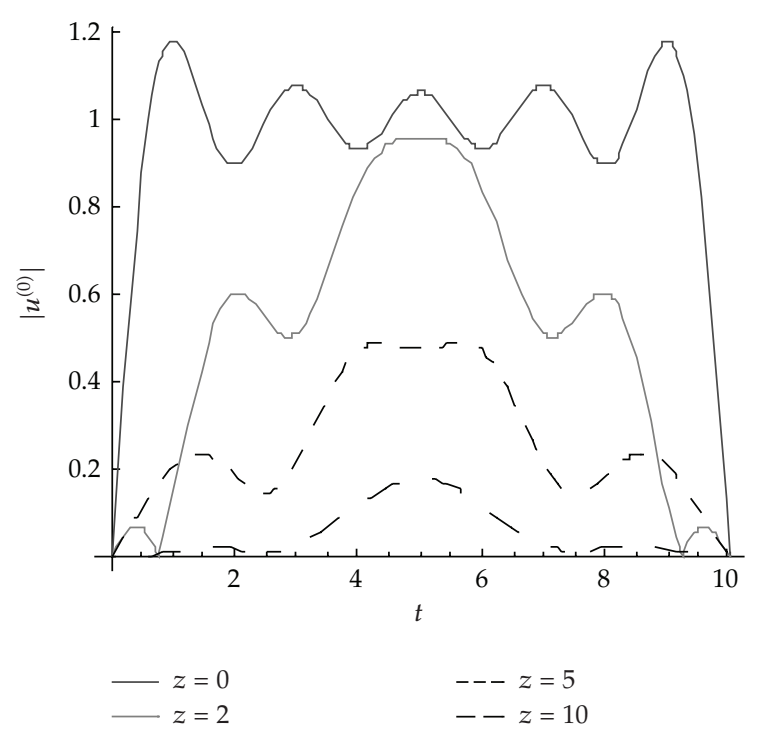

Figure 5: The zero-order approximation $\left|u^{(0)}\right|$ at $\alpha=1, T=10, \gamma=02$ for different values of $z$, considering only one term in the series $(M=1)$.

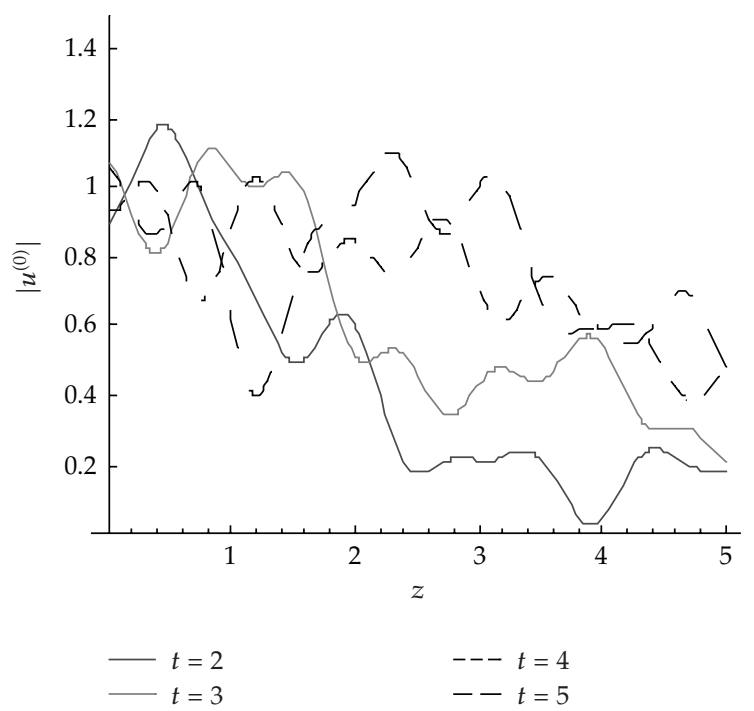

Figure 6: The zero-order approximation $\left|u^{(0)}\right|$ at $\alpha=1, T=10, \gamma=02$ for different values of $t$, considering only one term in the series $(M=1)$.

which has the solution, see the previous section,

$$
u_{0}(t, z)=e^{-\gamma z}\left(w_{0}(t, z)+i v_{0}(t, z)\right) .
$$




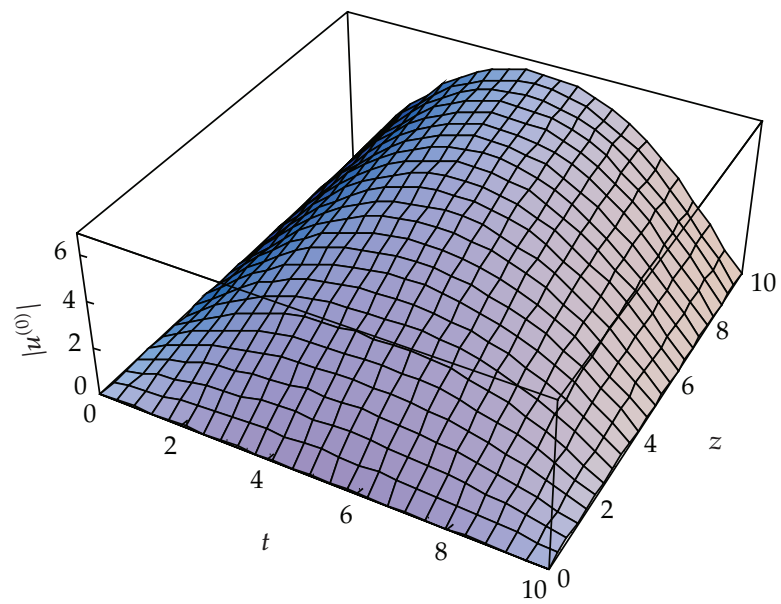

Figure 7: The zero-order approximation of $\left|u^{(0)}\right|$ at $\alpha=1, T=10, \gamma=02$ with considering only one term in the series $(M=1)$.

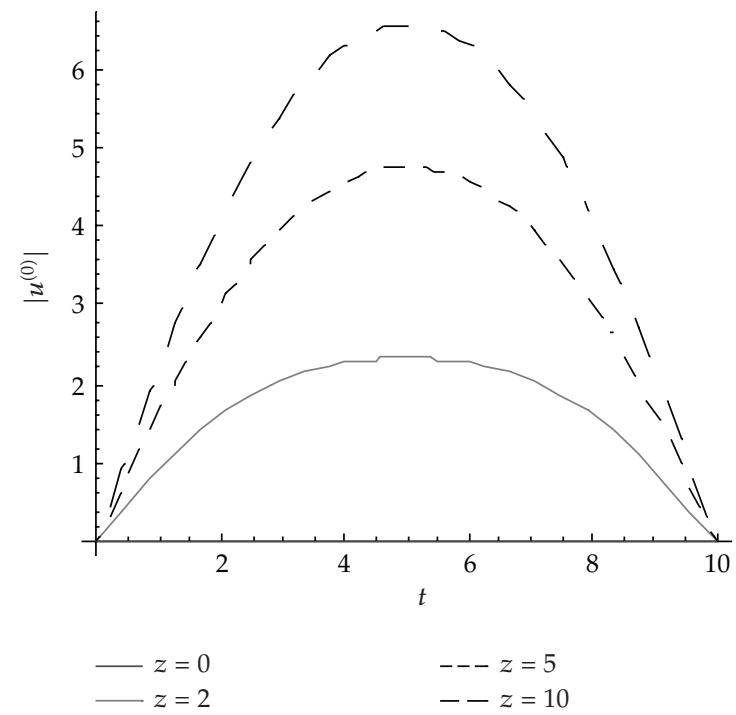

Figure 8: The zero-order approximation $\left|u^{(0)}\right|$ at $\alpha=1, T=10, \gamma=02$ for different values of $z$, considering only one term in the series $(M=1)$.

Following Pickard approximation, (3.13) can be rewritten as

$$
i \frac{\partial u_{n}(t, z)}{\partial z}+\alpha \frac{\partial^{2} u_{n}(t, z)}{\partial t^{2}}+i \gamma u_{n}(t, z)=F(t, z)-\varepsilon\left|u_{n-1}(t, z)\right|^{2} u_{n-1}(t, z), \quad n \geq 1
$$

At $n=1$, the iterative equation takes the following form:

$$
i \frac{\partial u_{1}(t, z)}{\partial z}+\alpha \frac{\partial^{2} u_{1}(t, z)}{\partial t^{2}}+i \gamma u_{1}(t, z)=F(t, z)-\varepsilon\left|u_{0}(t, z)\right|^{2} u_{0}(t, z)=F(t, z)+\varepsilon h_{1}(t, z)
$$




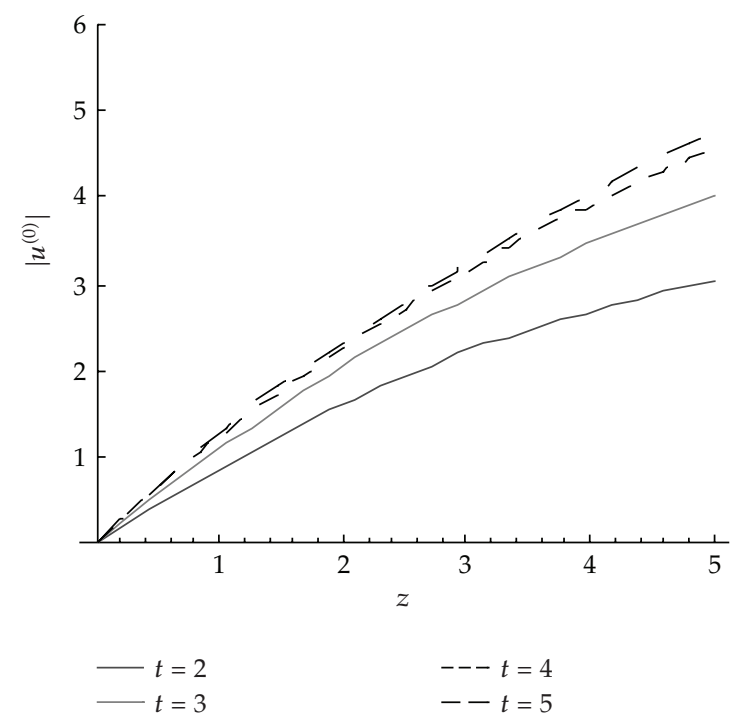

Figure 9: The zero-order approximation $\left|u^{(0)}\right|$ at $\alpha=1, T=10, \gamma=02$ for different values of $t$, considering only one term in the series $(M=1)$.

which can be solved as a linear case with zero initial and boundary conditions. The following general solution can be obtained:

$$
\begin{aligned}
w_{1}(t, z) & =\sum_{n=0}^{\infty}\left(T_{0 n}+\varepsilon T_{1 n}\right) \sin \left(\frac{n \pi}{T}\right) t \\
v_{1}(t, z) & =\sum_{n=0}^{\infty}\left(\tau_{0 n}+\varepsilon \tau_{1 n}\right) \sin \left(\frac{n \pi}{T}\right) t \\
u_{1}(t, z) & =e^{-\gamma z}\left(w_{1}(t, z)+i v_{1}(t, z)\right) \\
& =u_{1}^{(0)}+\varepsilon u_{1}^{(1)} .
\end{aligned}
$$

At $n=2$, the following equation is obtained:

$$
i \frac{\partial u_{2}(t, z)}{\partial z}+\alpha \frac{\partial^{2} u_{2}(t, z)}{\partial t^{2}}+i \gamma u_{2}(t, z)=F(t, z)-\varepsilon\left|u_{1}(t, z)\right|^{2} u_{1}(t, z)=F(t, z)+\varepsilon h_{2}(t, z)
$$

which can be solved as a linear case with zero initial and boundary conditions. The following general solution can be obtained:

$$
u_{2}(t, z)=u_{2}^{(0)}+\varepsilon u_{2}^{(1)}+\varepsilon^{2} u_{2}^{(2)}+\varepsilon^{3} u_{2}^{(3)}+\varepsilon^{4} u_{2}^{(4)}
$$

Continuing like this, one can get

$$
u_{n}(t, z)=u_{n}^{(0)}+\varepsilon u_{n}^{(1)}+\varepsilon^{2} u_{n}^{(2)}+\varepsilon^{3} u_{n}^{(3)}+\cdots+\varepsilon^{(n+m)} u_{n}^{(n+m)} .
$$




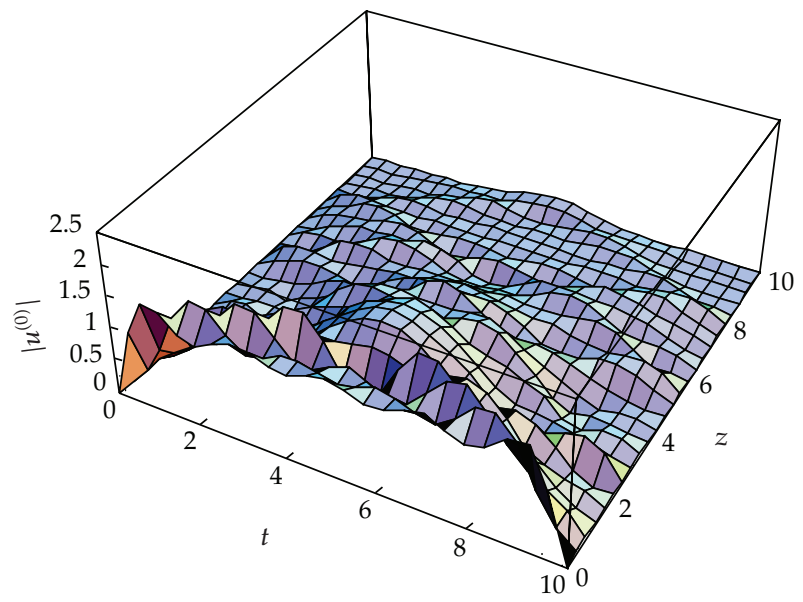

Figure 10: The zero-order approximation of $\left|u^{(0)}\right|$ at $\alpha=1, T=10, \gamma=02$ with considering only one term in the series $(M=1)$.

As $n \rightarrow \infty$, the solution (if exists) can be reached as $u(t, z)=\lim _{n \rightarrow \infty} u_{n}(t, z)$. Accordingly, the solution is a power series in $\varepsilon$.

According to the previous lemma, one can assume the solution of (3.1) as the following:

$$
u(t, z)=\sum_{n=0}^{\infty} \varepsilon^{n} u_{n}
$$

Let $u(t, z)=\psi(t, z)+i \phi(t, z), \psi, \phi$ : real valued functions. The following coupled equations are got:

$$
\begin{aligned}
& \frac{\partial \phi(t, z)}{\partial z}=\alpha \frac{\partial^{2} \psi(t, z)}{\partial t^{2}}+\varepsilon\left(\psi^{2}+\phi^{2}\right) \psi-\gamma \phi-F_{1} \\
& \frac{\partial \psi(t, z)}{\partial z}=-\alpha \frac{\partial^{2} \phi(t, z)}{\partial t^{2}}-\varepsilon\left(\psi^{2}+\phi^{2}\right) \phi-\gamma \psi+F_{2}
\end{aligned}
$$

where $\psi(t, 0)=f_{1}(t), \phi(t, 0)=f_{2}(t)$, and all corresponding other I.C. and B.C. are zeros.

As a perturbation solution, one can assume that

$$
\begin{aligned}
& \psi(t, z)=\psi_{0}+\varepsilon \psi_{1}+\varepsilon^{2} \psi_{2}+\cdots, \\
& \phi(t, z)=\phi_{0}+\varepsilon \phi_{1}+\varepsilon^{2} \phi_{2}+\cdots,
\end{aligned}
$$

where $\psi_{0}(t, 0)=f_{1}(t), \phi_{0}(t, 0)=f_{2}(t), \mathrm{k}$ and all corresponding other I.C. and B.C. are zeros. 
Substituting (3.12) into (3.11) and then equating the equal powers of $\varepsilon$, one can get the following set of coupled equations:

$$
\begin{aligned}
& \frac{\partial \phi_{0}(t, z)}{\partial z}=\alpha \frac{\partial^{2} \psi_{0}(t, z)}{\partial t^{2}}-\gamma \phi_{0}-F_{1} \\
& \frac{\partial \psi_{0}(t, z)}{\partial z}=-\alpha \frac{\partial^{2} \phi_{0}(t, z)}{\partial t^{2}}-\gamma \psi_{0}+F_{2} \\
& \frac{\partial \phi_{1}(t, z)}{\partial z}=\alpha \frac{\partial^{2} \psi_{1}(t, z)}{\partial t^{2}}-\gamma \phi_{1}+\left(\psi_{0}^{3}+\psi_{0} \phi_{0}^{2}\right) \\
& \frac{\partial \psi_{1}(t, z)}{\partial z}=-\alpha \frac{\partial^{2} \phi_{1}(t, z)}{\partial t^{2}}-\gamma \psi_{1}-\left(\phi_{0}^{3}+\phi_{0} \psi_{0}^{2}\right) \\
& \frac{\partial \phi_{2}(t, z)}{\partial z}=\alpha \frac{\partial^{2} \psi_{2}(t, z)}{\partial t^{2}}-\gamma \phi_{2}+\left(3 \psi_{0}^{2} \psi_{1}+2 \psi_{0} \phi_{0} \phi_{1}+\psi_{1} \phi_{0}^{2}\right) \\
& \frac{\partial \psi_{2}(t, z)}{\partial z}=-\alpha \frac{\partial^{2} \phi_{2}(t, z)}{\partial t^{2}}-\gamma \psi_{2}-\left(3 \phi_{0}^{2} \phi_{1}+2 \phi_{0} \psi_{0} \psi_{1}+\phi_{1} \psi_{0}^{2}\right)
\end{aligned}
$$

and so on. The prototype equations to be solved are

$$
\begin{aligned}
& \frac{\partial \phi_{i}(t, z)}{\partial z}=\alpha \frac{\partial^{2} \psi_{i}(t, z)}{\partial t^{2}}-\gamma \phi_{i}+G_{i}^{(1)}, \quad i \geq 1, \\
& \frac{\partial \psi_{i}(t, z)}{\partial z}=-\alpha \frac{\partial^{2} \phi_{i}(t, z)}{\partial t^{2}}-\gamma \psi_{i}+G_{i}^{(2)}, \quad i \geq 1,
\end{aligned}
$$

where $\psi_{i}(t, 0)=\delta_{i, 0} f_{1}(t), \phi_{i}(t, 0)=\delta_{i, 0} f_{2}(t)$ and all other corresponding conditions are zeros. The nonhomogeneity functions $G_{i}^{(1)}$ and $G_{i}^{(2)}$ are functions computed from previous steps.

Following the solution algorithm described in the previous section for the linear case, the general symbolic algorithm in Figure 39 can be simulated through the use of a symbolic package, mathematica- 5 is used in this paper.

\subsection{The Zero-Order Approximation}

In this case,

$$
u^{(0)}(t, z)=\left(\psi_{0}+i \phi_{0}\right)
$$




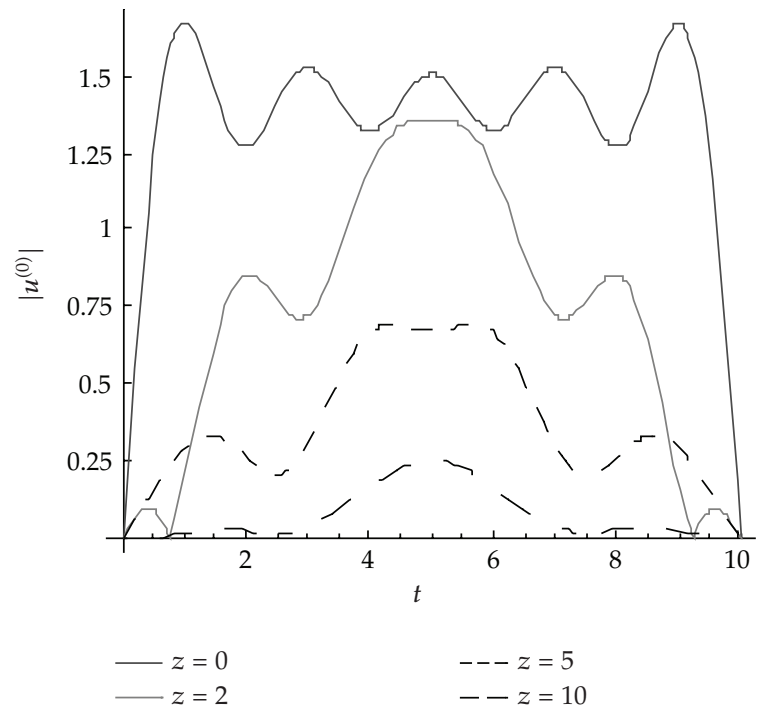

Figure 11: The zero-order approximation $\left|u^{(0)}\right|$ at $\alpha=1, T=10, \gamma=02$ for different values of $z$, considering only one term in the series $(M=1)$.

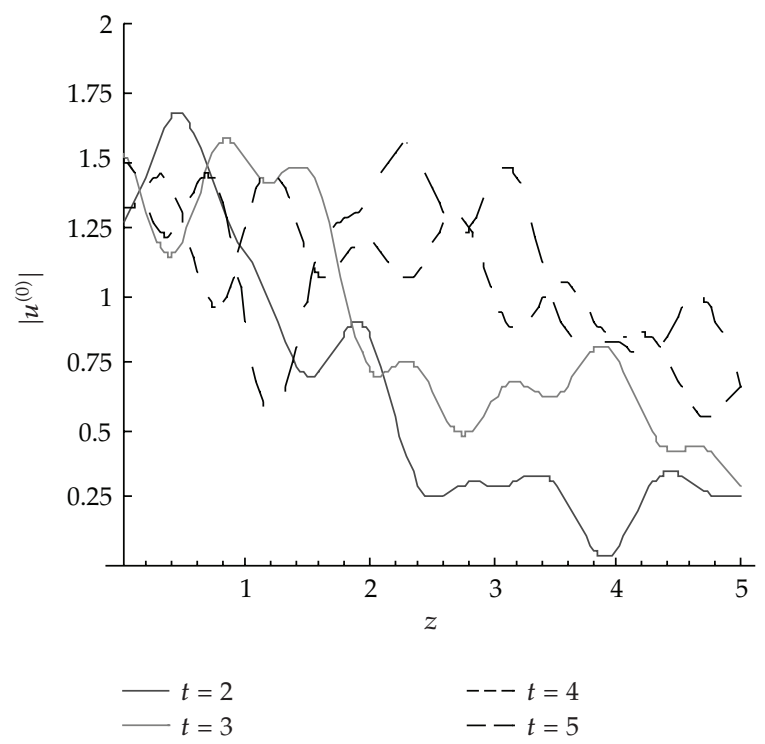

Figure 12: The zero-order approximation $\left|u^{(0)}\right|$ at $\alpha=1, T=10, \gamma=02$ for different values of $t$, considering only one term in the series $(M=1)$.

where

$$
\begin{aligned}
& \psi_{0}(t, z)=e^{-\gamma z} \sum_{n=0}^{\infty} T_{0 n} \sin \left(\frac{n \pi}{T}\right) t \\
& \phi_{0}(t, z)=e^{-\gamma z} \sum_{n=0}^{\infty} \tau_{0 n} \sin \left(\frac{n \pi}{T}\right) t
\end{aligned}
$$




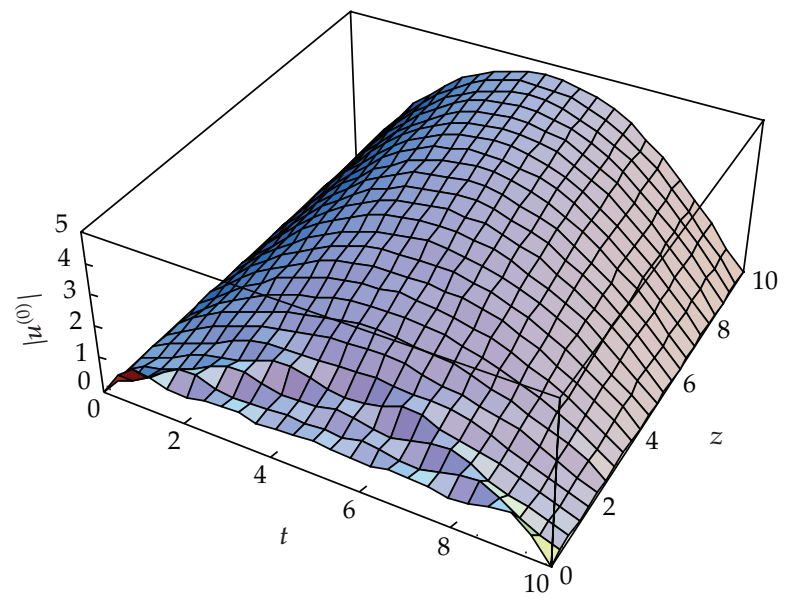

Figure 13: The zero-order approximation of $\left|u^{(0)}\right|$ at $\alpha=1, T=10, \gamma=02$ with considering only one term in the series $(M=1)$.

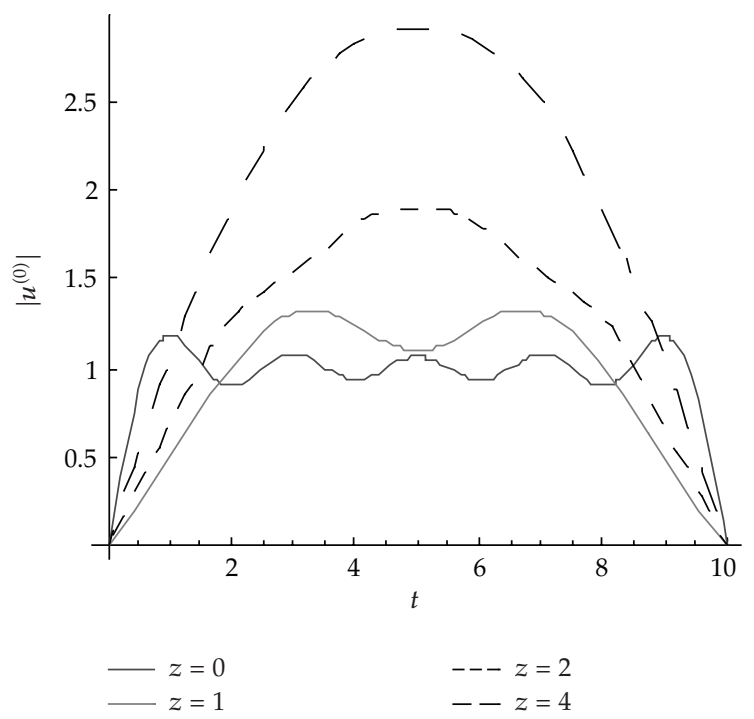

Figure 14: The zero-order approximation $\left|u^{(0)}\right|$ at $\alpha=1, T=10, \gamma=02$ for different values of $z$, considering only one term in the series $(M=1)$.

in which

$$
\begin{aligned}
& T_{0 n}(z)=A_{01}(z) \sin \beta_{n} z+\left(C_{02}+B_{01}(z)\right) \cos \beta_{n} z \\
& \tau_{0 n}(z)=A_{02}(z) \sin \beta_{n} z+\left(\widetilde{C}_{02}+B_{02}(z)\right) \cos \beta_{n} z
\end{aligned}
$$

where the constants and variables $A_{01}(z), C_{02}, B_{01}(z), A_{02}(z), \widetilde{C}_{02}$, and $B_{02}(z)$ can be got by the aid of Section 2. 


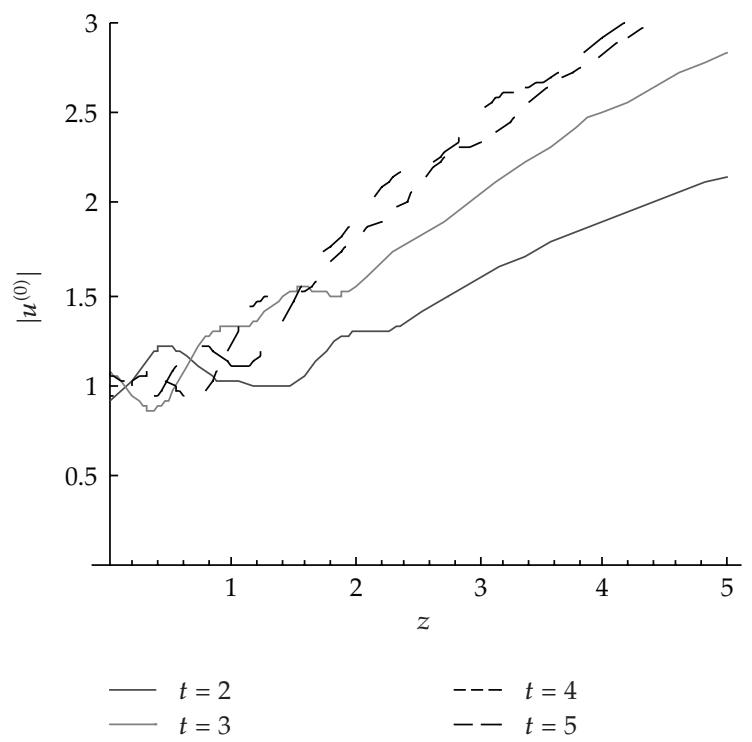

Figure 15: The zero-order approximation $\left|u^{(0)}\right|$ at $\alpha=1, T=10, \gamma=02$ for different values of $t$, considering only one term in the series $(M=1)$.

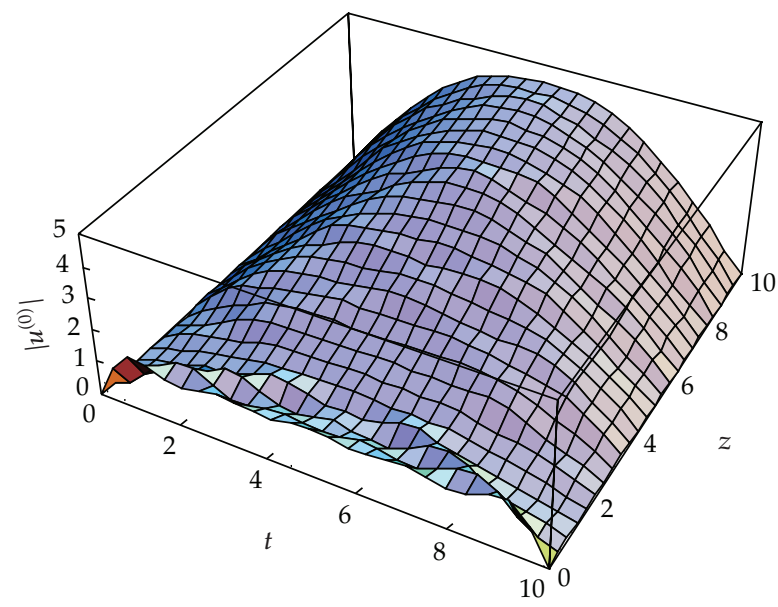

Figure 16: The zero-order approximation of $\left|u^{(0)}\right|$ at $\alpha=1, T=10, \gamma=02$ with considering only one term in the series $(M=1)$.

The absolute value of the zero-order approximation is got from

$$
\left|u^{(0)}(t, z)\right|^{2}=\psi_{0}{ }^{2}+\phi_{0}{ }^{2}
$$

\subsection{The First-Order Approximation}

$$
u^{(1)}(t, z)=u^{(0)}+\varepsilon\left(\psi_{1}+i \phi_{1}\right)
$$




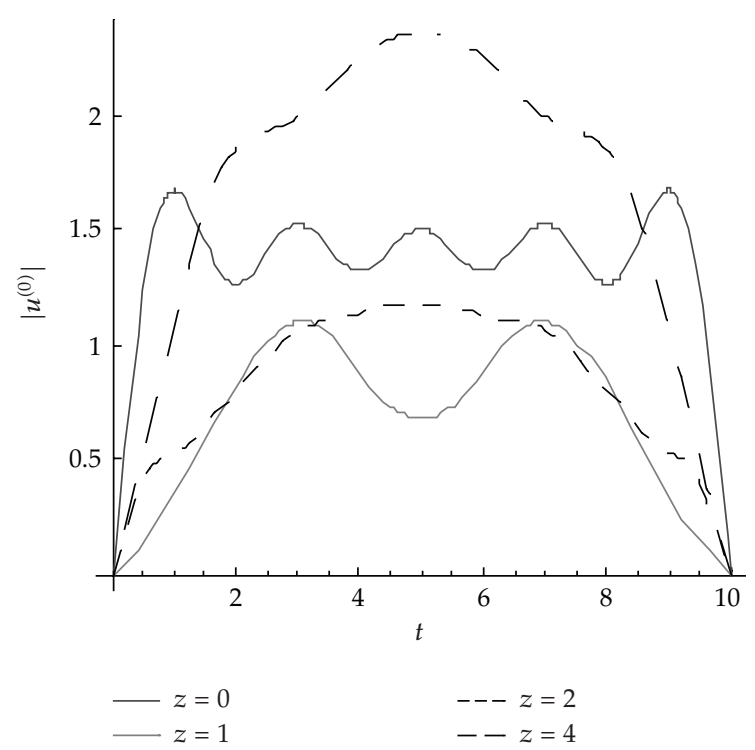

Figure 17: The zero-order approximation $\left|u^{(0)}\right|$ at $\alpha=1, T=10, \gamma=02$ for different values of $z$, considering only one term in the series $(M=1)$.

where

$$
\begin{aligned}
& \psi_{1}(t, z)=e^{-\gamma z} \sum_{n=0}^{\infty} T_{1 n}(z) \sin \left(\frac{n \pi}{T}\right) t \\
& \phi_{1}(t, z)=e^{-\gamma z} \sum_{n=0}^{\infty} \tau_{1 n}(z) \sin \left(\frac{n \pi}{T}\right) t
\end{aligned}
$$

in which

$$
\begin{aligned}
& T_{1 n}(z)=A_{11}(z) \sin \beta_{n} z+\left(C_{12}+B_{11}(z)\right) \cos \beta_{n} z \\
& \tau_{1 n}(z)=A_{12}(z) \sin \beta_{n} z+\left(\widetilde{C}_{12}+B_{12}(z)\right) \cos \beta_{n} z
\end{aligned}
$$

where the constants and variables $A_{11}(z), B_{11}(z), A_{12}(z)$, and $B_{12}(z)$ can be evaluated in a similar manner as the zero-order approximation whereas $\widetilde{C}_{12}=-B_{12}(0)$ and $C_{12}=-B_{11}(0)$.

The absolute value of the first-order approximation can be got using

$$
\left|u^{(1)}(t, z)\right|^{2}=\left|u^{(0)}(t, z)\right|^{2}+2 \varepsilon\left(\psi_{0} \psi_{1}+\phi_{0} \phi_{1}\right)+\varepsilon^{2}\left(\psi_{1}{ }^{2}+\phi_{1}{ }^{2}\right)
$$

\subsection{The Second-Order Approximation}

$$
u^{(2)}(t, z)=u^{(1)}(t, z)+\varepsilon^{2}\left(\psi_{2}+i \phi_{2}\right)
$$




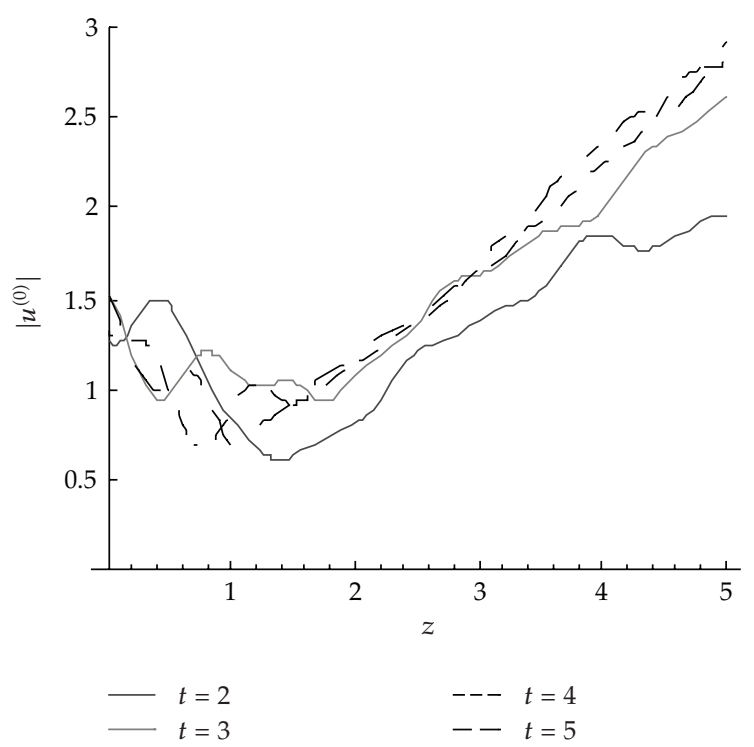

Figure 18: The zero-order approximation $\left|u^{(0)}\right|$ at $\alpha=1, T=10, \gamma=02$ for different values of $t$, considering only one term in the series $(M=1)$.

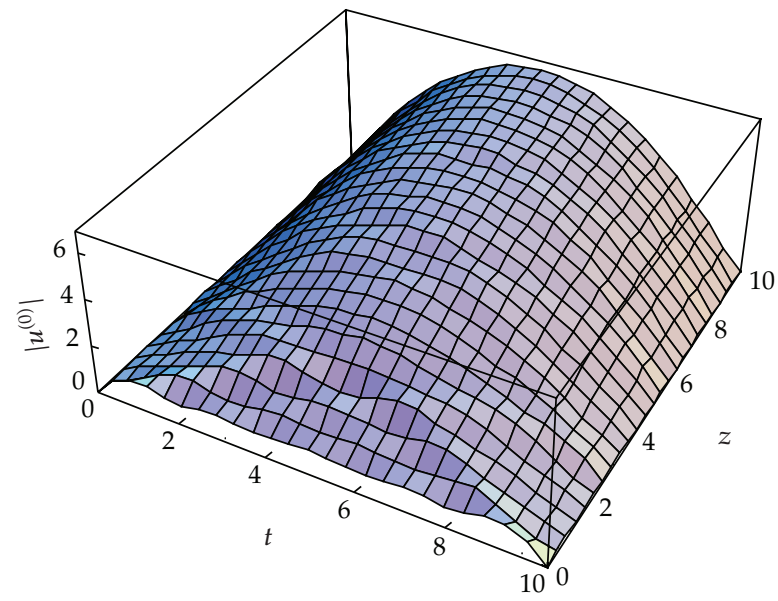

Figure 19: The zero-order approximation of $\left|u^{(0)}\right|$ at $\alpha=1, T=10, \gamma=02$ with considering only one term in the series $(M=1)$.

where

$$
\begin{aligned}
& \psi_{2}(t, z)=e^{-\gamma z} \sum_{n=0}^{\infty} T_{2 n}(z) \sin \left(\frac{n \pi}{T}\right) t \\
& \phi_{2}(t, z)=e^{-\gamma z} \sum_{n=0}^{\infty} \tau_{2 n}(z) \sin \left(\frac{n \pi}{T}\right) t
\end{aligned}
$$




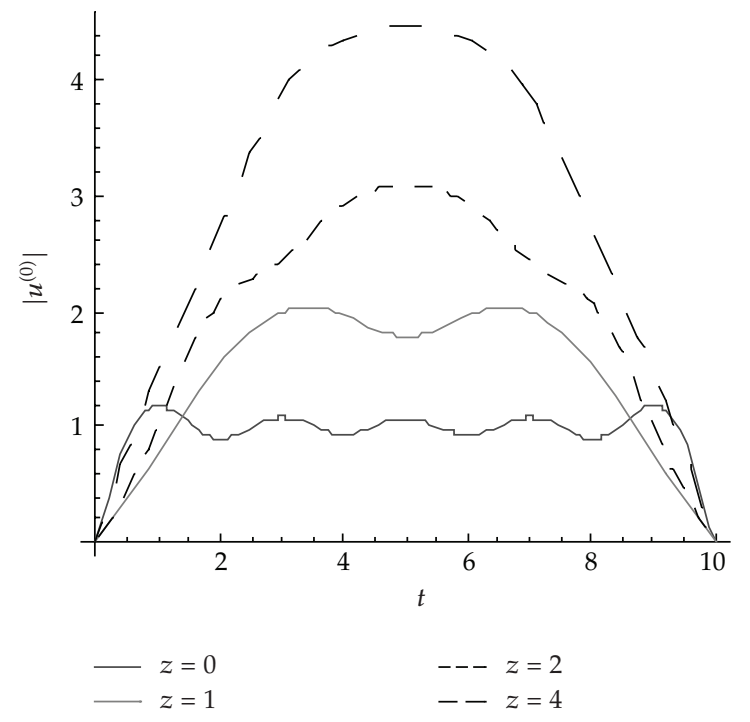

Figure 20: The zero-order approximation $\left|u^{(0)}\right|$ at $\alpha=1, T=10, \gamma=02$ for different values of $z$, considering only one term in the series $(M=1)$.

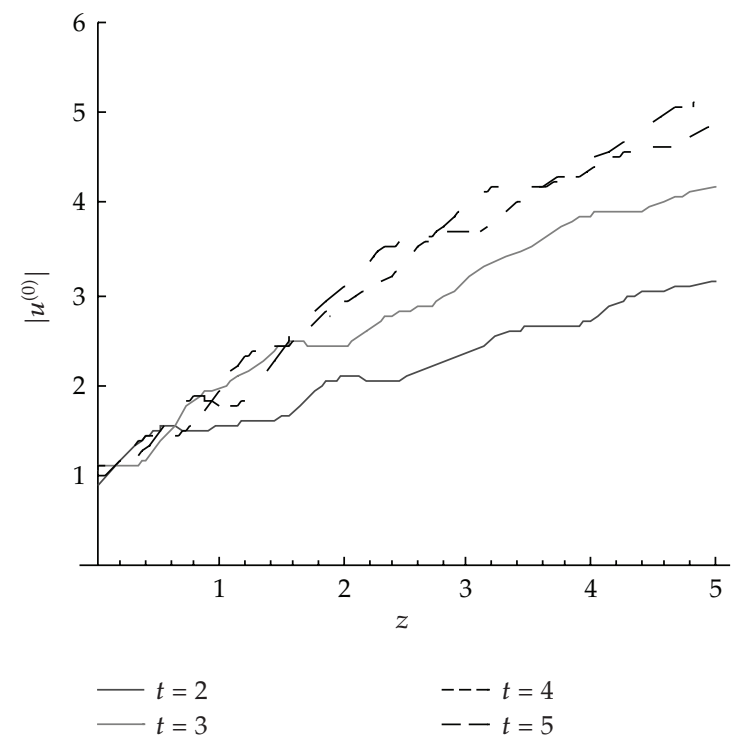

Figure 21: The zero-order approximation $\left|u^{(0)}\right|$ at $\alpha=1, T=10, \gamma=02$ for different values of $t$, considering only one term in the series $(M=1)$.

in which

$$
\begin{aligned}
& T_{2 n}(z)=A_{21}(z) \sin \beta_{n} z+\left(C_{22}+B_{21}(z)\right) \cos \beta_{n} z \\
& \tau_{2 n}(z)=A_{22}(z) \sin \beta_{n} z+\left(\widetilde{C}_{22}+B_{22}(z)\right) \cos \beta_{n} z
\end{aligned}
$$




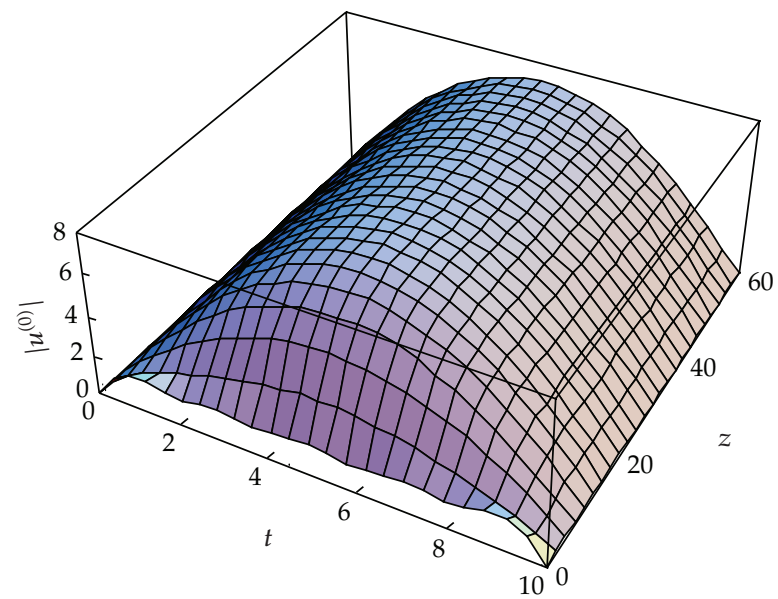

Figure 22: The zero-order approximation of $\left|u^{(0)}\right|$ at $\alpha=1, T=10, \gamma=02$ with considering only one term in the series $(M=1)$.

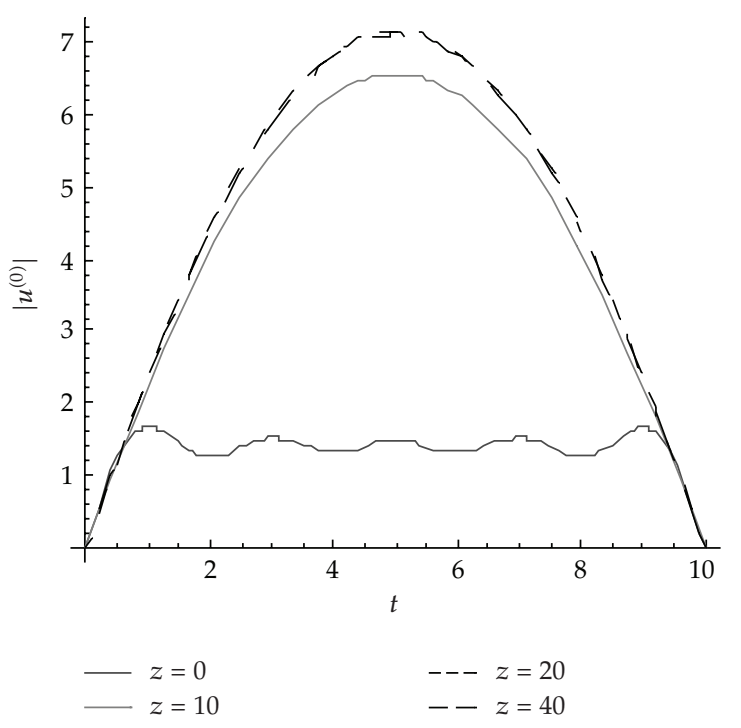

Figure 23: The zero-order approximation $\left|u^{(0)}\right|$ at $\alpha=1, T=10, \gamma=02$ for different values of $z$, considering only one term in the series $(M=1)$.

where the constants and variables $A_{21}(z), C_{22}, B_{21}(z), A_{22}(z), \widetilde{C}_{22}$, and $B_{22}(z)$ can be evaluated similarly as the previous approximation.

The absolute value of the second-order approximation can be got using

$$
\left|u^{(2)}(t, z)\right|^{2}=\left|u^{(1)}(t, z)\right|^{2}+2 \varepsilon^{2}\left(\psi_{0} \psi_{2}+\phi_{0} \phi_{2}\right)+2 \varepsilon^{3}\left(\psi_{1} \psi_{2}+\phi_{1} \phi_{2}\right)+\varepsilon^{4}\left(\psi_{2}^{2}+\phi_{2}^{2}\right) .
$$

\section{Case Studies}

To examine the proposed solution algorithm, see Figure 39, some case studies are illustrated. 


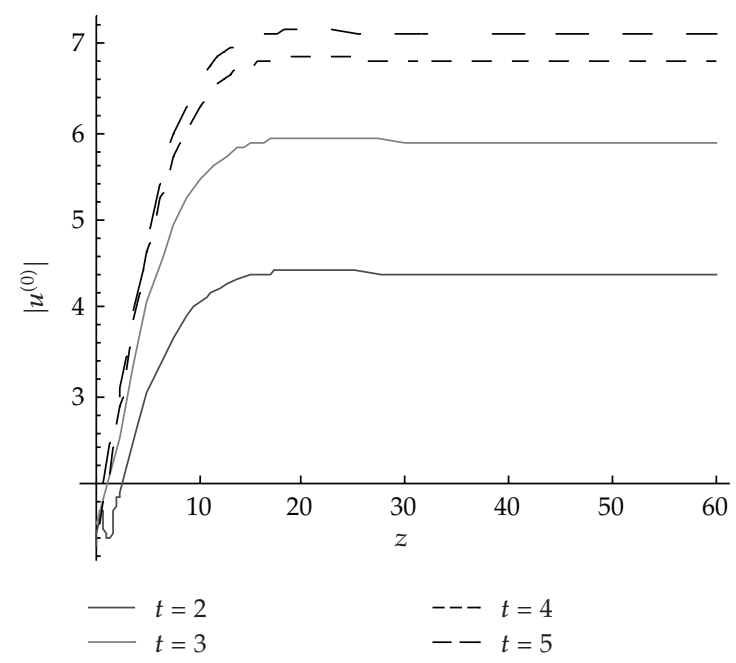

Figure 24: The zero-order approximation $\left|u^{(0)}\right|$ at $\alpha=1, T=10, \gamma=02$ for different values of $t$, considering only one term in the series $(M=1)$.

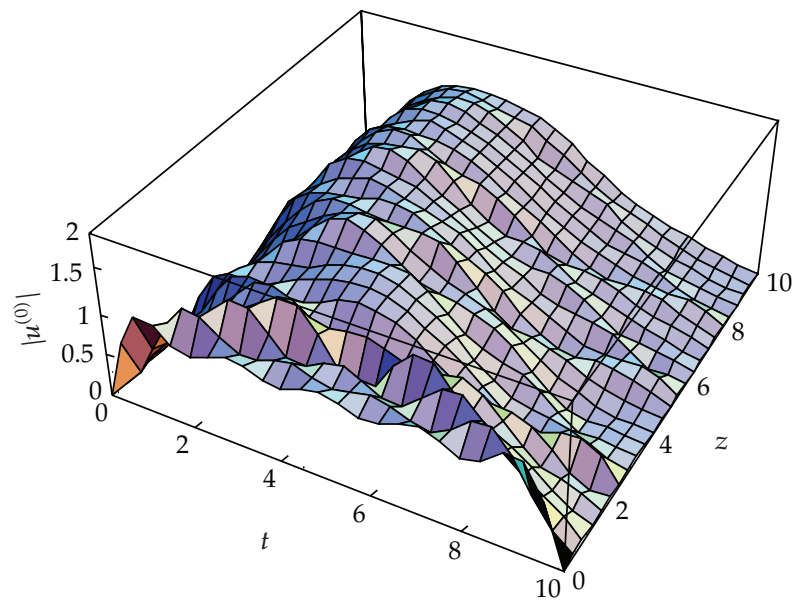

Figure 25: The zero-order approximation of $\left|u^{(0)}\right|$ at $\alpha=1, T=10, \gamma=02$ with considering only one term in the series $(M=1)$.

\subsection{One Input Is On}

\section{Case Study 1}

Taking $f_{1}(t)=0, f_{2}(t)=0, F_{1}(t, z)=1, F_{2}(t, z)=0$, and following the solution algorithm, the selective results for the zero-order approximation are got in Figures 1, 2, and 3.

\section{Case Study 2}

Taking $f_{1}(t)=0, f_{2}(t)=0, F_{1}(t, z)=0, F_{2}(t, z)=1$ and following the solution algorithm, it has been noticed that the same results for the case study 1 are got. 


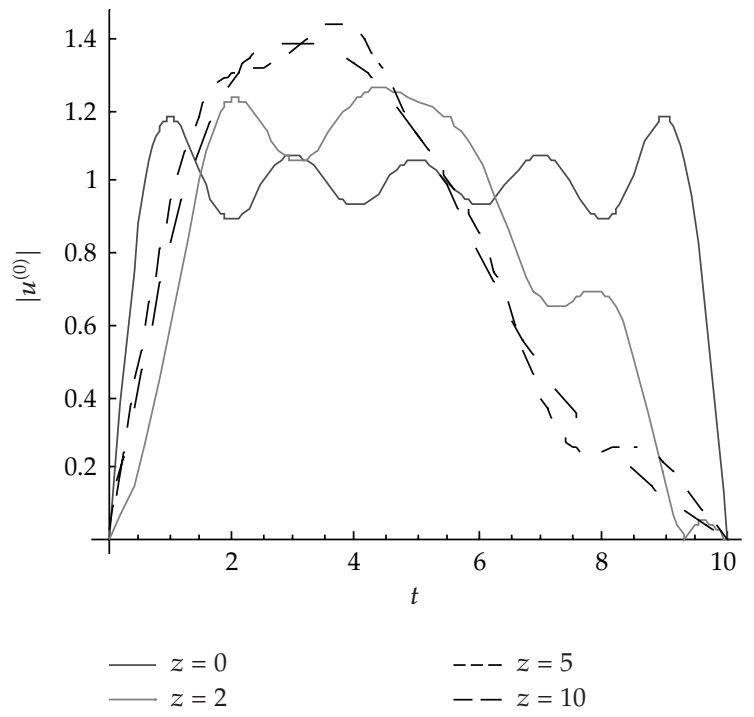

Figure 26: The zero-order approximation $\left|u^{(0)}\right|$ at $\alpha=1, T=10, \gamma=02$ for different values of $z$, considering only one term in the series $(M=1)$.

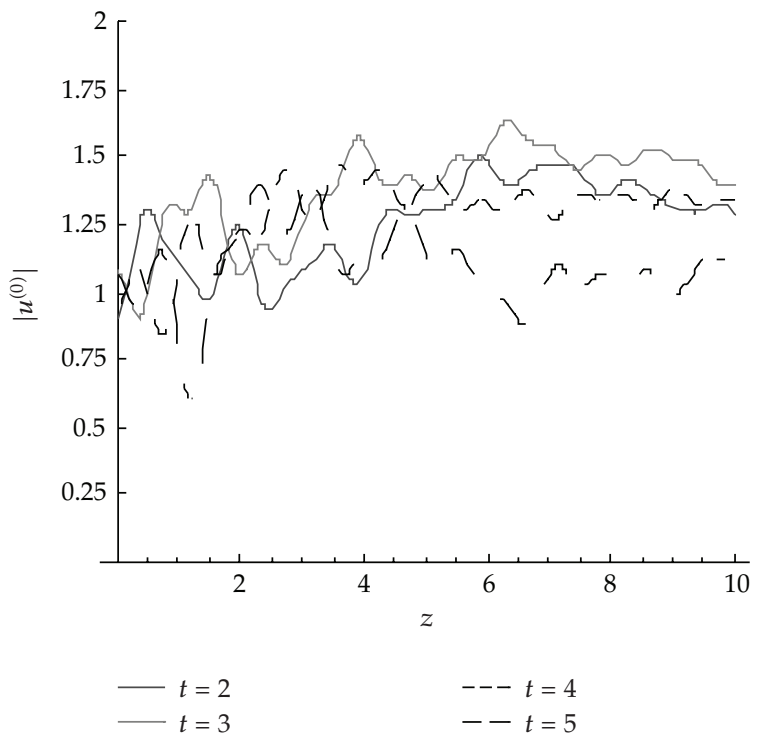

Figure 27: The zero-order approximation $\left|u^{(0)}\right|$ at $\alpha=1, T=10, \gamma=02$ for different values of $t$, considering only one term in the series $(M=1)$.

\section{Case Study 3}

Taking $f_{1}(t)=1, f_{2}(t)=0, F_{1}(t, z)=0, F_{2}(t, z)=0$ and following the solution algorithm, the selective results for the first-zero approximation are got in Figures 4, 5, and 6.

One can notice the decrease of the solution level and its higher variability. 


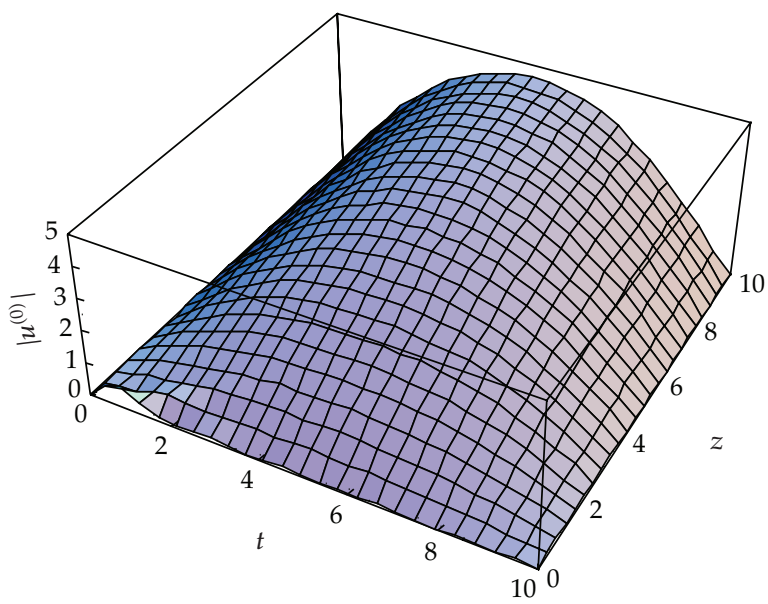

Figure 28: The zero-order approximation of $\left|u^{(0)}\right|$ at $\alpha=1, T=10, \gamma=02$ with considering only one term in the series $(M=1)$.

\section{Case Study 4}

Taking $f_{1}(t)=0, f_{2}(t)=1, F_{1}(t, z)=0, F_{2}(t, z)=0$ and following the solution algorithm, it has been noticed that the same results for the case study 3 are got:

\subsection{Two Inputs Are On}

\section{Case Study 5}

Taking $f_{1}(t)=0, f_{2}(t)=0, F_{1}(t, z)=1, F_{2}(t, z)=1$ and following the solution algorithm, the selective results for the zero-order approximation are got in Figures 7, 8, and 9.

One can notice that the solution level becomes a little bit higher than that of case study 2 .

\section{Case Study 6}

Taking $f_{1}(t)=1, f_{2}(t)=1, F_{1}(t, z)=0, F_{2}(t, z)=0$ and following the solution algorithm, the selective results for the zero-order approximation are got in Figures 10, 11, and 12.

One can notice the little increase of the solution level than that of case studies 3 and 4 .

\section{Case Study 7}

Taking $f_{1}(t)=1, f_{2}(t)=0, F_{1}(t, z)=1, F_{2}(t, z)=0$ and following the solution algorithm, the selective results for the zero-order approximation are got in Figures 13, 14, and 15.

One can notice the small perturbations at small values of $z$. 


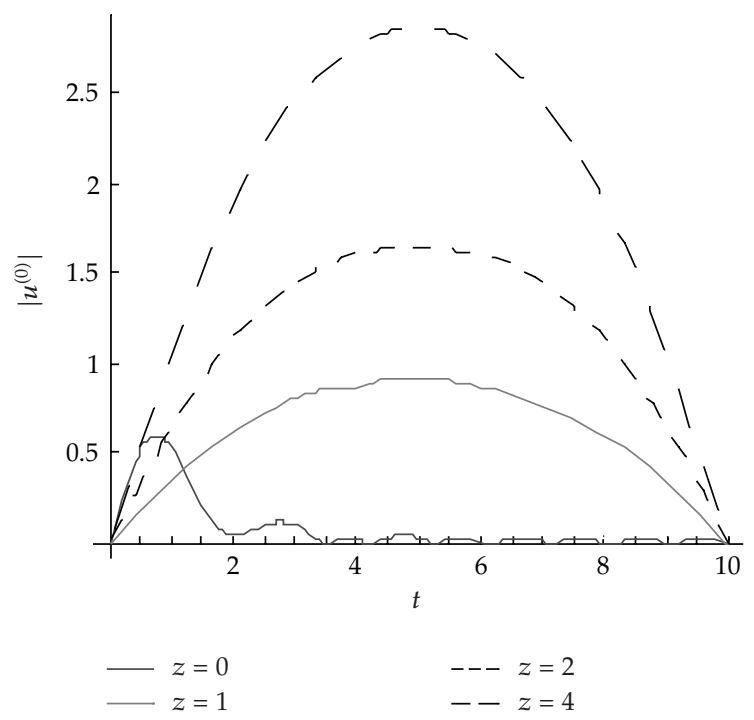

Figure 29: The zero-order approximation $\left|u^{(0)}\right|$ at $\alpha=1, T=10, \gamma=02$ for different values of $z$, considering only one term in the series $(M=1)$.

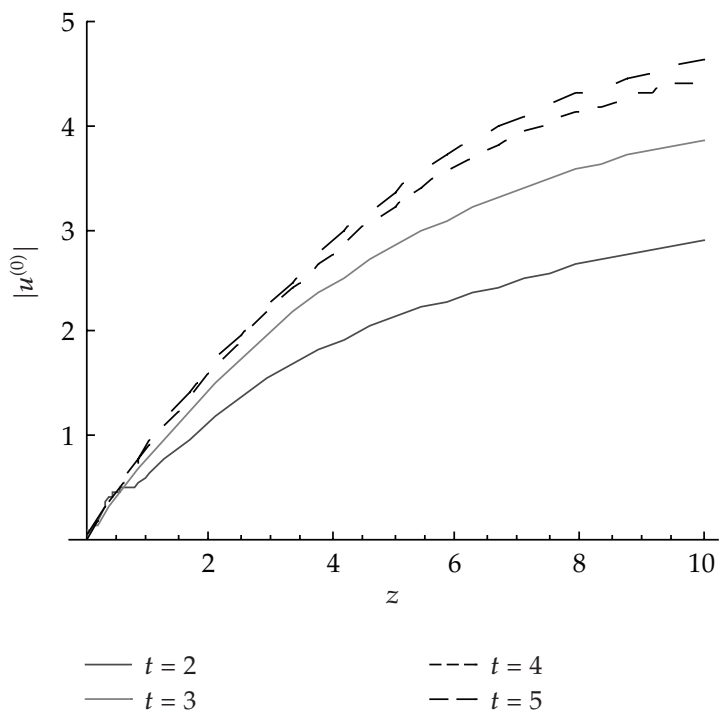

Figure 30: The zero-order approximation $\left|u^{(0)}\right|$ at $\alpha=1, T=10, \gamma=02$ for different values of $t$, considering only one term in the series $(M=1)$.

\subsection{Three Inputs Are On}

Case Study 8

Taking $f_{1}(t)=1, f_{2}(t)=1, F_{1}(t, z)=1, F_{2}(t, z)=0$ and following the solution algorithm, the selective results for the zero-order approximation are got in Figures 16, 17, and 18.

One can notice the increase of the depth of the perturbations. 


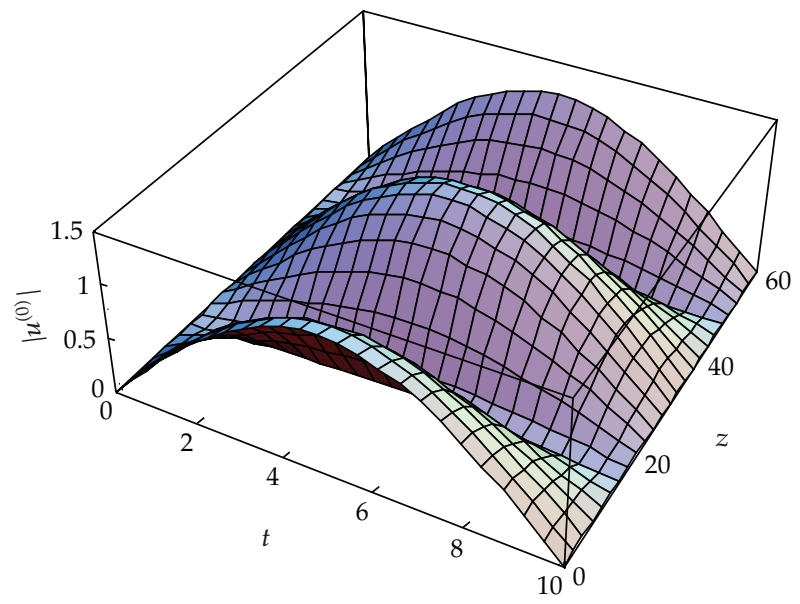

Figure 31: The zero-order approximation of $\left|u^{(0)}\right|$ at $\alpha=1, T=10, \gamma=0$ with considering only one term in the series $(M=1)$.

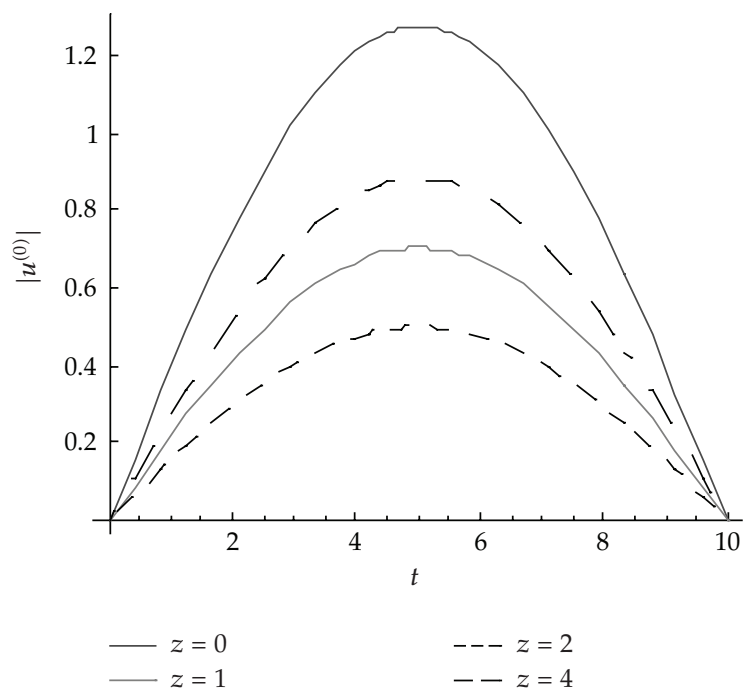

Figure 32: The zero-order approximation $\left|u^{(0)}\right|$ at $\alpha=1, T=10, \gamma=0$ for different values of $z$, considering only one term in the series $(M=1)$.

\section{Case Study 9}

Taking $f_{1}(t)=1, f_{2}(t)=0, F_{1}(t, z)=1, F_{2}(t, z)=1$ and following the solution algorithm, the selective results for the zero-order approximation are got in Figures 19, 20, and 21.

One can notice that the perturbations become smaller than that of case study 8 . 


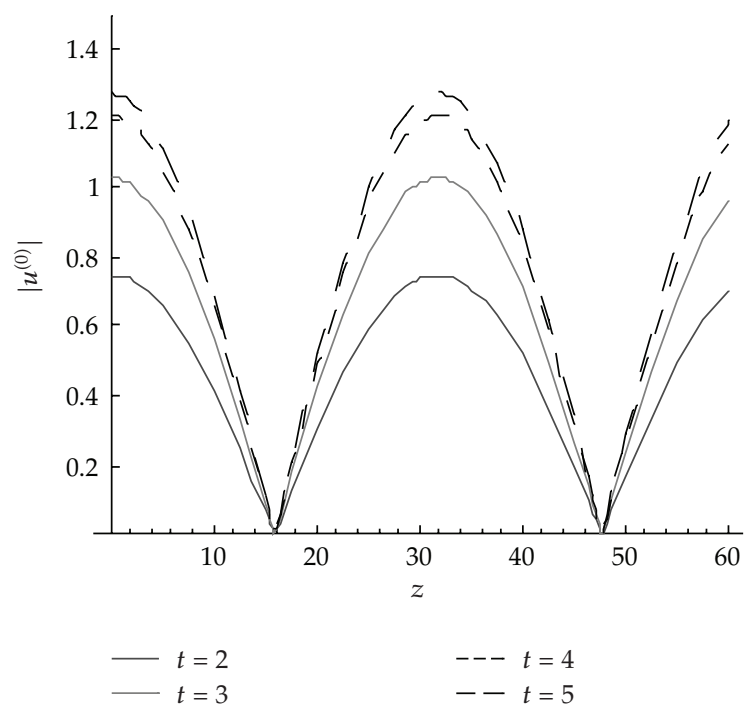

Figure 33: The zero-order approximation $\left|u^{(0)}\right|$ at $\alpha=1, T=10, \gamma=0$ for different values of $t$, considering only one term in the series $(M=1)$.

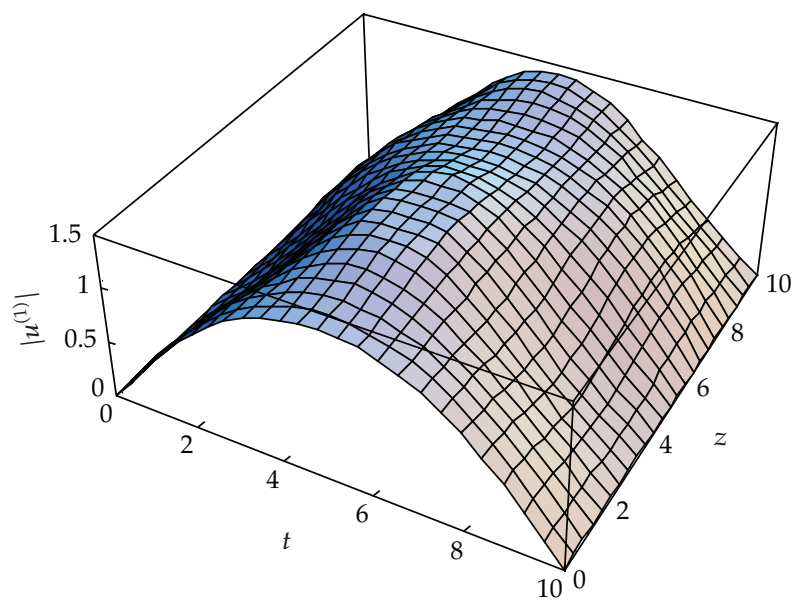

Figure 34: The first-order approximation of $\left|u^{(1)}\right|$ at $\alpha=1, T=10, \gamma=0, \varepsilon=01$ with considering only one term in the series $(M=1)$.

\subsection{Four Inputs Are On}

Case Study 10

Taking the case of $f_{1}(t)=1, f_{2}(t)=1, F_{1}=1, F_{2}=1$, the following final results for the zero-order approximation are obtained in Figures 22, 23, and 24.

One can notice the little increase in the solution level. 


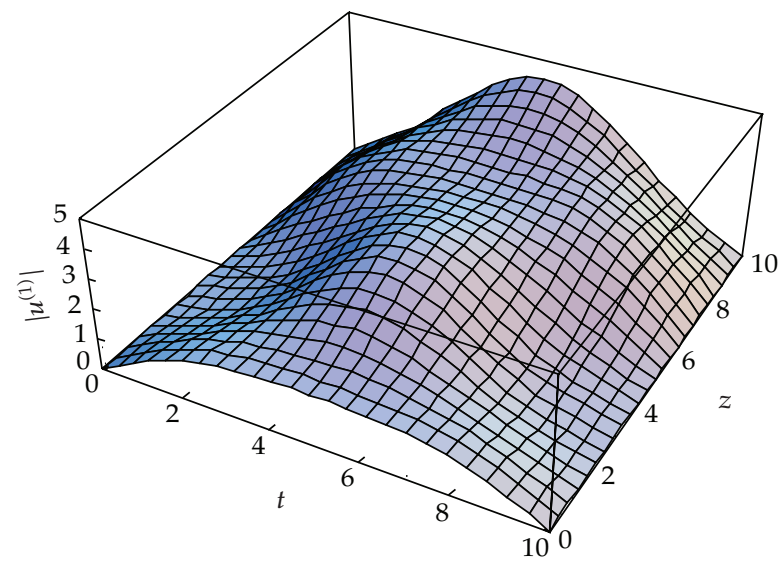

Figure 35: The first-order approximation of $\left|u^{(1)}\right|$ at $\alpha=1, T=10, \gamma=0, \varepsilon=05$ with considering only one term in the series $(M=1)$.

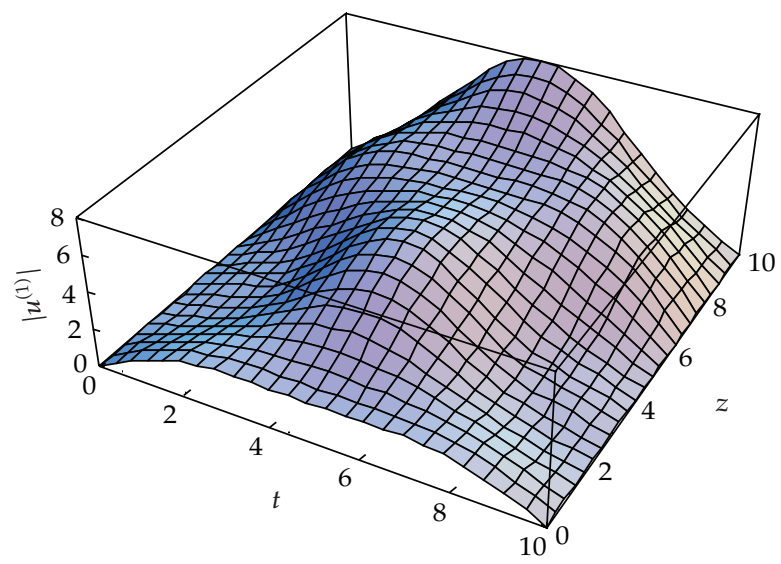

Figure 36: The first-order approximation of $\left|u^{(1)}\right|$ at $\alpha=1, T=10, \gamma=0, \varepsilon=1$ with considering only one term in the series $(M=1)$.

\subsection{Exponential Nonhomogeneity}

\section{Case Study 11}

Taking the case of $f_{1}(t)=1, f_{2}(t)=0, F_{1}(t, z)=e^{-t}, F_{2}(t, z)=0$, the following final results for the zero-order approximation are obtained in Figures 25, 26, and 27.

One can notice the low solution level and high perturbations. 


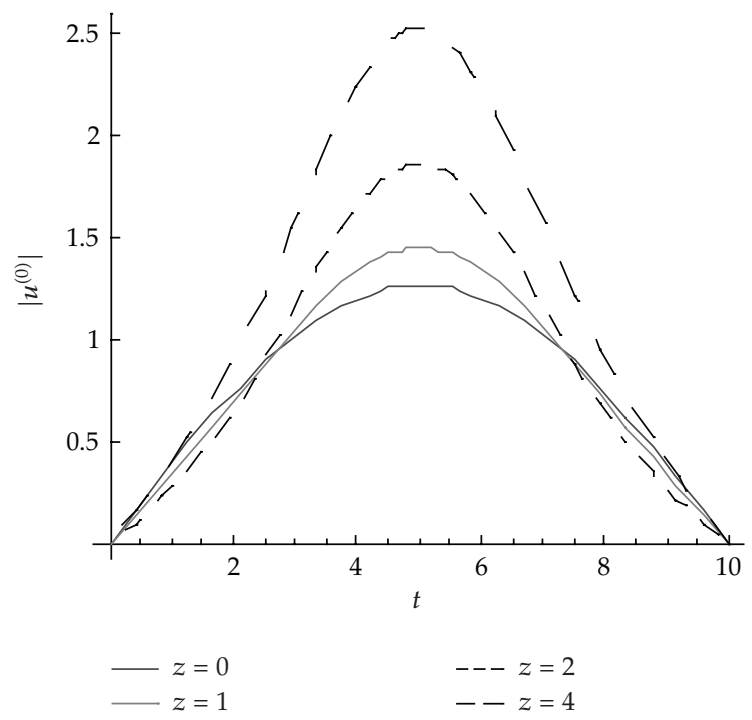

Figure 37: The first-order approximation $\left|u^{(1)}\right|$ at $\alpha=1, T=10, \gamma=0, \varepsilon=05$ for different values of $z$, considering only one term in the series $(M=1)$.

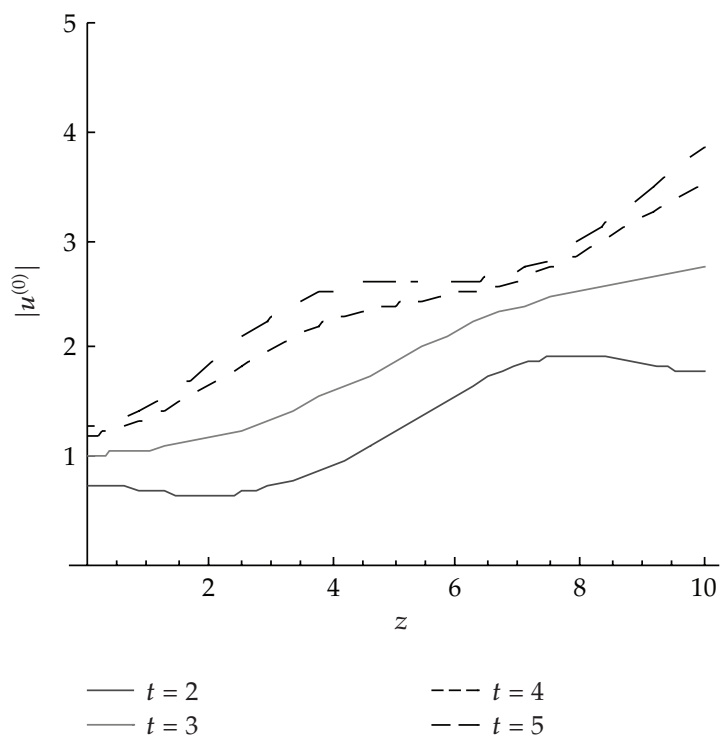

Figure 38: The first-order approximation $\left|u^{(1)}\right|$ at $\alpha=1, T=10, \gamma=0, \varepsilon=05$ for different values of $t$, considering only one term in the series $(M=1)$.

\subsection{Exponential Initial Condition}

Case Study 12

Taking the case of $f_{1}(t)=e^{-t}, f_{2}(t)=0, F_{1}(t, z)=1, F_{2}(t, z)=0$, the following final results for the zero-order approximation are obtained in Figures 28, 29, and 30. 


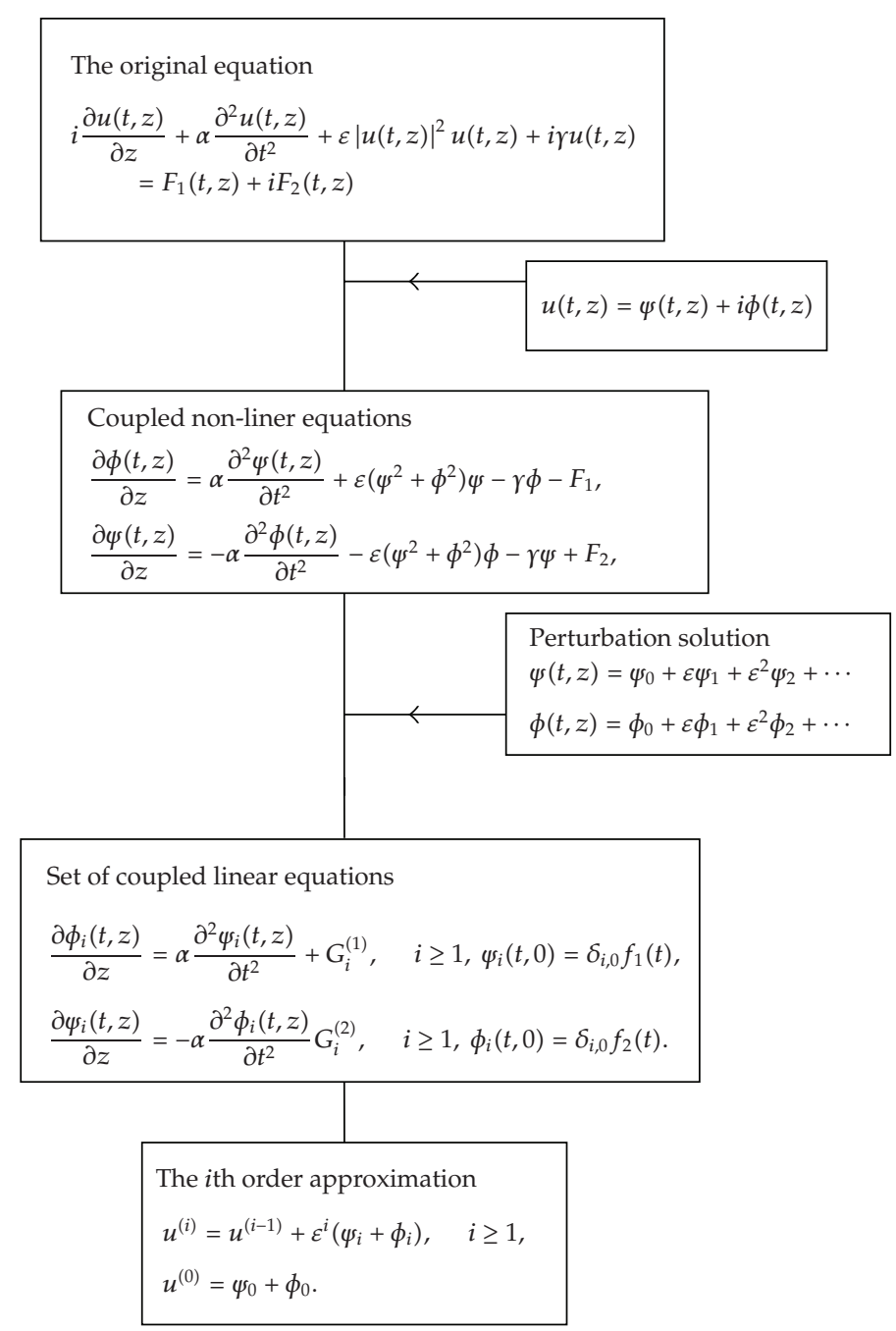

Figure 39: The general solution algorithm.

One can notice that a higher solution level is got compared with case study 11 and less perturbations are got at small values of $z$.

\subsection{First-Order Approximation}

Case Study 13

Taking the case of $f_{1}(t)=1, f_{2}(t)=0, F_{1}(t, z)=1, F_{2}(t, z)=0$, the following final results for the zero and first-order approximations are obtained in Figures 31-38.

One can notice the oscillations of the solution level compared with case 7.

One can notice that the solution level increases with the increase of $\varepsilon$. 


\section{Conclusions}

The perturbation technique introduces an approximate solution to the NLS equation with a perturbative nonlinear term for a finite time interval. Using mathematica, the difficult and huge computations problems were fronted to some extent for limited series terms. To get more improved orders, it is expected to face a problem of computation. With respect to the solution level, the effect of the nonhomogeneity is higher than the effect of the initial condition. The initial conditions also cause perturbations for the solution at small values of the space variable. The solution level increases with the increase of $\varepsilon$.

\section{References}

[1] M. J. Ablowitz, B. M. Herbst, and C. M. Schober, "The nonlinear Schrödinger equation: asymmetric perturbations, traveling waves and chaotic structures," Mathematics and Computers in Simulation, vol. 43, no. 1, pp. 3-12, 1997.

[2] F. Kh. Abdullaev, J. C. Bronski, and G. Papanicolaou, "Soliton perturbations and the random Kepler problem," Physica D, vol. 135, no. 3-4, pp. 369-386, 2000.

[3] S. Fewo, J. Atangana, A. Kenfack-Jiotsa, and T. C. Kofane, "Dispersion-managed solitons in the cubic complex Ginzburg-Landau equation as perturbations of nonlinear Schrodinger equation," Optics Communications, vol. 252, pp. 138-149, 2005.

[4] A. Biswas and K. Porsezian, "Soliton perturbation theory for the modified nonlinear Schrödinger's equation," Communications in Nonlinear Science and Numerical Simulation, vol. 12, no. 6, pp. 886-903, 2007.

[5] T. Cazenave and P.-L. Lions, “Orbital stability of standing waves for some nonlinear Schrödinger equations," Communications in Mathematical Physics, vol. 85, no. 4, pp. 549-561, 1982.

[6] A. Debussche and L. Di Menza, "Numerical simulation of focusing stochastic nonlinear Schrödinger equations," Physica D, vol. 162, no. 3-4, pp. 131-154, 2002.

[7] A. Debussche and L. Di Menza, "Numerical resolution of stochastic focusing NLS equations," Applied Mathematics Letters, vol. 15, no. 6, pp. 661-669, 2002.

[8] M. Wang, X. Li, and J. Zhang, "Various exact solutions of nonlinear Schrödinger equation with two nonlinear terms," Chaos, Solitons and Fractals, vol. 31, no. 3, pp. 594-601, 2007.

[9] L.-P. Xu and J.-L. Zhang, "Exact solutions to two higher order nonlinear Schrödinger equations," Chaos, Solitons and Fractals, vol. 31, no. 4, pp. 937-942, 2007.

[10] N. H. Sweilam, "Variational iteration method for solving cubic nonlinear Schrödinger equation," Journal of Computational and Applied Mathematics, vol. 207, no. 1, pp. 155-163, 2007.

[11] S.-D. Zhu, "Exact solutions for the high-order dispersive cubic-quintic nonlinear Schrödinger equation by the extended hyperbolic auxiliary equation method," Chaos, Solitons and Fractals, vol. 34, no. 5, pp. 1608-1612, 2007.

[12] J.-Q. Sun, Z.-Q. Ma, W. Hua, and M.-Z. Qin, "New conservation schemes for the nonlinear Schrödinger equation," Applied Mathematics and Computation, vol. 177, no. 1, pp. 446-451, 2006.

[13] K. Porsezian and B. Kalithasan, "Cnoidal and solitary wave solutions of the coupled higher order nonlinear Schrödinger equation in nonlinear optics," Chaos, Solitons and Fractals, vol. 31, no. 1, pp. 188-196, 2007.

[14] H. Sakaguchi and T. Higashiuchi, "Two-dimensional dark soliton in the nonlinear Schrödinger equation," Physics Letters A, vol. 359, no. 6, pp. 647-651, 2006.

[15] D.-J. Huang, D.-S. Li, and H.-Q. Zhang, "Explicit and exact travelling wave solutions for the generalized derivative Schrödinger equation," Chaos, Solitons and Fractals, vol. 31, no. 3, pp. 586-593, 2007.

[16] W. K. Abou Salem and C. Sulem, "Stochastic acceleration of solitons for the nonlinear Schrödinger equation," SIAM Journal on Mathematical Analysis, vol. 41, no. 1, pp. 117-152, 2009.

[17] M. El-Tawil, "The average solution of a stochastic nonlinear Schrodinger equation under stochastic complex non-homogeneity and complex initial conditions," in Transactions on Computational Science III, vol. 5300 of Lecture Notes in Computer Science, pp. 143-170, Springer, New York, NY, USA, 2009.

[18] M. Colin, T. Colin, and Mohta, "Stability of solitary eaves for a system of nonlinear Schrodinger equations with three wave interactions," to appear in Annals de I'Institut Henri Poincare (c) Nonlinear Analysis. 
[19] Z. Jia-Min and L. Yu-Lu, "Some exact solutions of variable coefficient cubic quintic nonlinear Schrodinger equation with an external potential," Communications in Theoretical Physics, vol. 51, no. 3, p. 391, 2009.

[20] S. J. Farlow, Partial Differential Equations for Scientists and Engineers, John Wiley \& Sons, New York, NY, USA, 1982.

[21] L. Pipes and L. Harvill, Applied Mathematics for Engineers and Physicists, McGraw-Hill, Tokyo, Japan, 1970. 


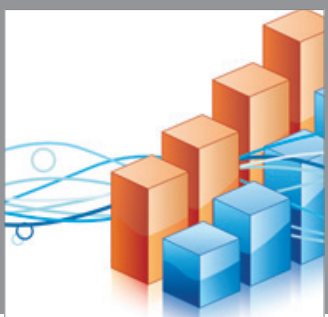

Advances in

Operations Research

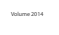

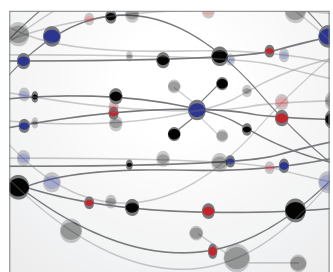

\section{The Scientific} World Journal
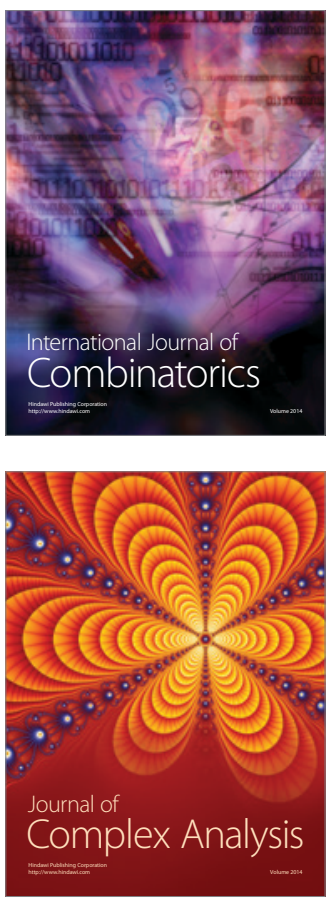

International Journal of

Mathematics and

Mathematical

Sciences
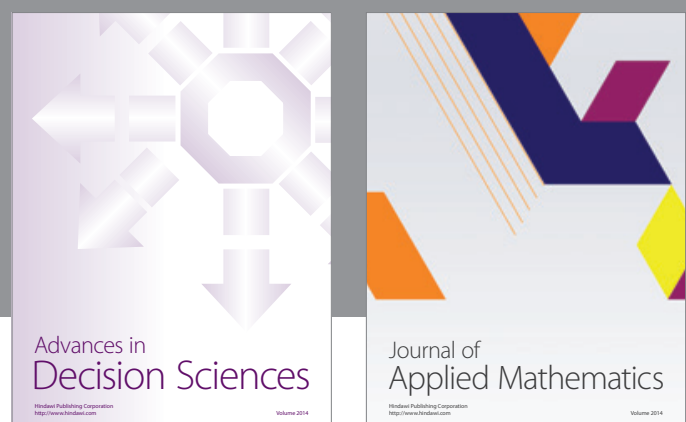

Journal of

Applied Mathematics
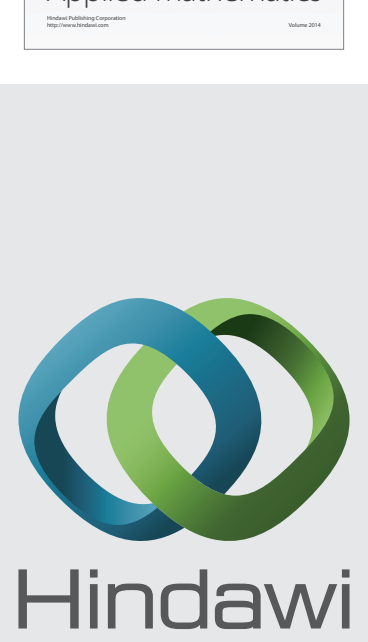

Submit your manuscripts at http://www.hindawi.com
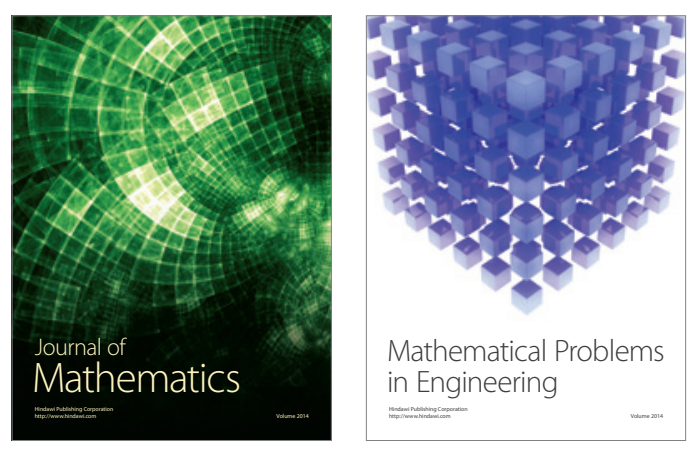

Mathematical Problems in Engineering
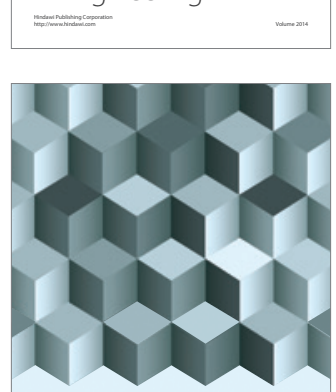

Journal of

Function Spaces
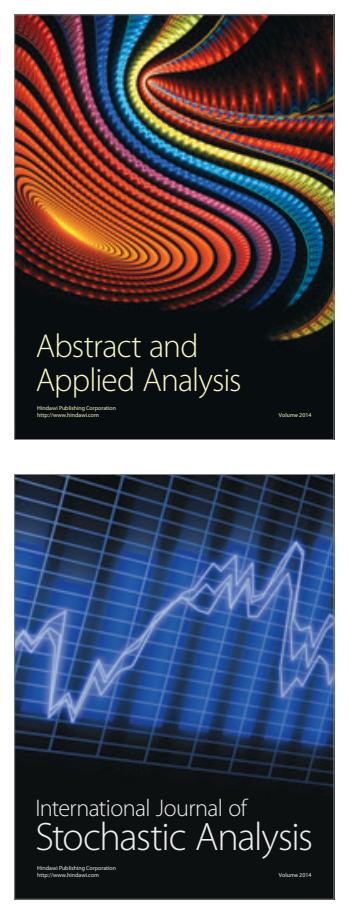

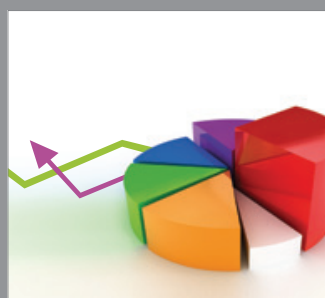

ournal of

Probability and Statistics

Promensencen
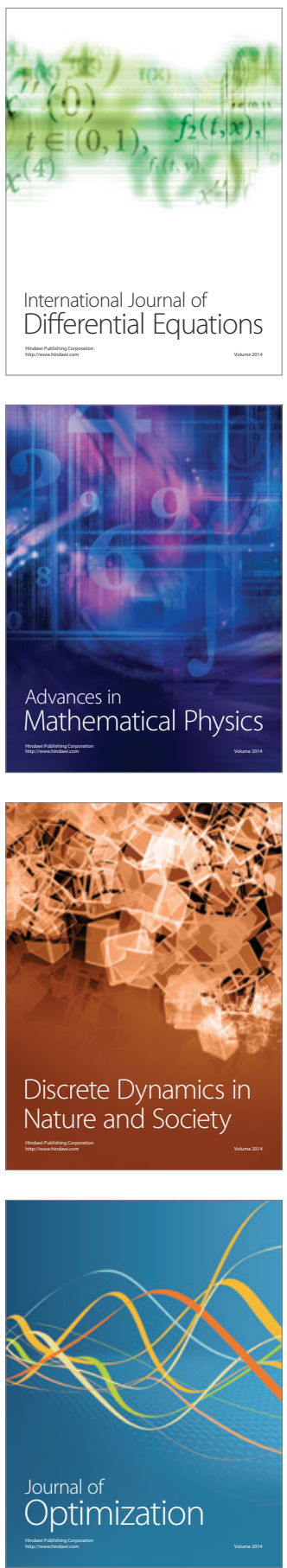